\title{
6 Der komödische Modus und die Dynamik des Genres
}

\subsection{Auftakt}

In düsterer Gestimmtheit betritt der Dirigent den Konzertsaal. Wie wird er das Orchester führen, wie die Pausen aushalten, die leisen, ruhigen Akzente und romantische Motive spielen, da er doch innerlich von tiefem Groll eingenommen ist? Die Komödie UNFAITHFULLY YOURS ${ }^{1}$ ergründet diesen Zusammenhang von affektiver Stimmung, künstlerischer Kreativität und Genre als Vorstellungsbilder des Verdachts und Wahns. Das misstrauische Phantasieren des Dirigenten über die Untreue seiner Frau findet dreimal nacheinander, in drei verschiedenen Stimmungen, statt. Während des Dirigierens dreier ganz unterschiedlicher Stücke malt er sich verschiedene Varianten des Ehebruchs aus. Dreimal stimmt er ein neues Stück an und dreimal hat es eine andere Tonalität, mit der vom Konzertsaal in die jeweilige Phantasie überblendet wird. Dabei sind es keine Rück- oder Vorblenden, sondern gedankliche Hypothesen, Möglichkeitsformen und Phantasmen, welche für den Zuschauer erfahrbar werden. Alle drei Szenarien des Ehebruchs sind von derselben Ausgangssituation bestimmt, die jeweils in ihrer Grundstimmung durch den unterschiedlichen musikalischen Ausdruck gewandelt wird: kaltblütig-düster, komisch-gelassen und melodramatisch-unglücklich. $\mathrm{Zu}$ Rossinis Ouvertüre von Semiramide, einer ,Oper seria‘, wird ein Mord phantasiert, das Verbrechen ist dabei in das abgründige chiaroscuro des Film noir getaucht; beim Präludium aus Wagners romantischer Oper Tannhäuser herrscht hingegen unbeschwerte Großzügigkeit: Der Betrogene lässt seine Frau ziehen und sorgt sogar noch für ihr finanzielles Auskommen; während Tschaikowskis dunkler Liebessymphonie Francesca da Rimini schließlich wandelt sich die Ausweglosigkeit der Situation in die Vision eines Selbstmords des Dirigenten. UNFAITHFULLY YOURS reflektiert, wie künstlerische (musikalische) Inszenierungen durch ihre Zeitgestaltung Affekte hervorbringen; wie durch die Tempi und Rhythmen der Tonfolgen, Harmonien und instrumentalen Klangfarben sich Gefühlsformationen ausbilden und wie diese höchst unterschiedliche Imaginationen als emotionale Szenarien entwerfen. Die Gefühlswandlungen sind zwar zum Künstlerego narrativiert, aber man durchläuft als Zuschauer die immer gleich beginnende Handlungsabfolge der verdächtigten Ehefrau in den unterschiedlichen Empfindungen bzw. Genreinszenierungen.

1 UNFAITHFULLY YOURS (Preston Sturges, USA 1948).

¿ Open Access. (c) 2020 Sarah Greifenstein, publiziert von De Gruyter. (cc) BY-NC-ND Dieses Werk ist lizenziert unter der Creative Commons Attribution-NonCommercial-NoDerivatives 4.0 Lizenz.

https://doi.org/10.1515/9783110519662-006 
In diesem Kapitel steht die Screwball Comedy als Genre im Zentrum. Während in den letzten drei Kapiteln der Schwerpunkt auf Mikroanalysen lag, welche die Affektpoetiken der Filme an drei paradigmatischen Inszenierungsformen untersuchten (Stimmbild, Paargeste, kommunizierendes Gesicht), geht es nun um eine Perspektive, die das gesamte Genre beleuchtet. Dabei frage ich nach dem spezifischen komödischen Modus der Inszenierung, welche die Screwball Comedies ausbilden. Dieser Modus ist in der Inszenierung begründet, lässt sich aber auch als Gefühlston beschreiben und ist zeitlich $\mathrm{zu}$ bestimmen. In diesem $\mathrm{Zu}$ sammenhang werden zunächst Theoriebezüge von Zeitlichkeit und Komödie erörtert. Dabei verbinde ich drei Zugänge (Quintilian, Bergson, Langer) mit den bisherigen Perspektiven der Arbeit (6.2). Im Anschluss daran wird eine dynamische Auffassung von Gattung entworfen, welche Genrefilme als ,familienähnlich* begreift in ihrer kinematografischen Gestaltung von Gefühl, Erfahrung und Denken, was sich über die Zeit transformiert (6.3.). Die Familienähnlichkeiten lassen sich an Szenentypen sowie an poetologischen Prinzipien festmachen (6.4.).

\subsection{Die Zeitform der Komödie}

Welche Elemente, Prinzipien und Charakteristika bilden die Poetologien der Schrewball Komödien aus? Im Zusammenhang mit diesen Fragen sind zwei Perspektiven von Bedeutung. Einerseits mag man erörtern, was sich das Genre der Screwball Comedy mit anderen Komödientraditionen verbindet, wie es sich reflexiv auf vergangene Poetologien, literarische oder filmische Strömungen bezieht, wie es dramatische Prinzipien des Komödienhaften aus Theatertraditionen aufnimmt. Andererseits wäre zu ergründen, wie sich das Genre in seiner historischkulturellen Konstellation spezifiziert hat, wie sich in den Filmen ein gewisser Zeitgeist, bestimmte Konzepte von Gesellschaft und Individuum, von Geschlechterverhältnissen und sozialen Werten manifestieren. In der zweiten Ausrichtung ginge es darum, festzustellen, worüber man in einer ganz bestimmten historischen Situation gelacht hat, welche Tabus bestanden, welche Auffassungen von Liebe, Ehe und Sexualität, welche gesellschaftlichen Thematiken in und mit den Komödien reflektiert wurden. Letzteres spricht vor allem eine Vorstellung von Film an, die in der bisherigen Untersuchung angelegt ist und in diesem Kapitel weiterverfolgt werden soll: dass nämlich Filme selbst - über die Produktion und Aufführung hinaus - in ihrer Rezeption als kulturelle Praktiken zu verstehen sind, denen Muster der Wahrnehmung, der Gefühlslenkung und des Denkens eingeschrieben sind, die im Modus des Filme-Sehens und -Hörens auf einen geteilten Horizont (im kleinsten gemeinsamen Nenner ist dies die geteilte filmische Wahrnehmung) verweisen und den sich Zuschauer aktiv, jeweils eingebettet in 
ihre historisch-kulturelle sowie mediale und individuelle Disposition, konstruktiv aneignen. ${ }^{2}$ Damit ist eine Perspektive angesprochen, in der sich die Geschichte der Medien und Kunst als eine Geschichte der Wahrnehmungsformen ausprägt, die sich in ästhetischen Praktiken nachzeichnen lässt. Diese sehr grundlegende Vorstellung spricht Walter Benjamin im Kunstwerkaufsatz an:

Innerhalb großer geschichtlicher Zeiträume verändert sich mit der gesamten Daseinsweise der menschlichen Kollektiva auch die Art und Weise ihrer Sinneswahrnehmung. Die Art und Weise, in der die menschliche Sinneswahrnehmung sich organisiert - das Medium, in dem sie erfolgt - ist nicht nur natürlich, sondern auch geschichtlich bedingt. Die Zeit der Völkerwanderung, in der die spätrömische Kunstindustrie und die Wiener Genesis entstanden, hatte nicht nur eine andere Kunst als die Antike, sondern auch eine andere Wahrnehmung. ${ }^{3}$

Es sind die Kunstwerke, Relikte, Bauten und musealen Objekte, an denen man eine bestimmte Geschichtlichkeit der Wahrnehmung dingfest machen kann. In dieser Hinsicht sind Filme weder Abbilder gesellschaftlicher Realität, noch sind sie einfach nur fiktive Narrationen, die $\mathrm{zu}$ einem bestimmten Zeitpunkt ein Publikum amüsierten. Vielmehr begründeten sie zur Zeit ihrer Aufführung aber auch heute aus der Retrospektive selbst jeweils neue Wahrnehmungszusammenhänge, Gefühle, Gedanken und Vorstellungen in den Einstellungen ihrer Zuschauer. Die Filme bringen eine ganz bestimmte Anordnung der Bewegungsbilder als Wahrnehmungsmodus mit sich, der dann im Filme-Sehen und -Hören von Zuschauerinnen im Akt der Aneignung neu konstruiert wird, wobei die Anordnung als auch die Aneignung selbst Teil einer Historizität von Wahrnehmung, von Empfinden und Denken ist. ${ }^{4}$ Erst in diesem Sinn kann man sagen, dass man, indem man den ästhetischen Erfahrungsmodus von Filmen beschreibt, eine Kulturgeschichte von Perzepten, Affekten und Denkformen rekonstruiert. ${ }^{5}$ Genre ist für meine Untersuchung als Begriff eine Möglichkeit, diese Anordnung über den einzelnen Film hinaus nachzuvollziehen.

Im Zusammenhang mit Genre frage ich aus der Perspektive einer Geschichte der Wahrnehmung nach den Formen der Affizierung, nach dem komödischen Modus. Es fällt auf, dass seit den antiken Konzepten zu Humor, Komödie, Komik und Lachen immer wieder eine Vorstellung aufgetaucht ist, welche sich mit den komischen Phänomenen verbindet: die Idee, dass der Erheiterung eine soziale oder individuelle Ventil- oder Regelfunktion innewohne. Der Gedanke spiegelt sich in den drei Begriffen, der Überlegenheit, Entlastung und

2 Kappelhoff 2018, z.B. S. 17.

3 Walter Benjamin: Das Kunstwerk im Zeitalter seiner technischen Reproduzierbarkeit (1936/ 1939). In: ders.: Medienästhetische Schriften. Frankfurt a. M. 2002, S. 351-383. S. 356.

4 Zum Konzept des Filme-Sehens sowie dem Begriff der Aneignung siehe Kappelhoff 2018 S. 9-18.

5 Siehe Kappelhoff 2018; Kappelhoff 2004a; Deleuze 1997 (1983); Deleuze 1997 (1985). 
Inkongruenz $z^{6}$ wider. Meist werden die Terme als konkurrierende Thesen formuliert. Man kann sie jedoch auch aufeinander beziehen.

1) Ein Beispiel für die erste These der Überlegenheit ist die berühmte Auffassung Henri Bergsons vom Lachen als soziale Geste. ${ }^{7}$ Das Lachen wird dabei als Akt der Ausgrenzung verstanden; im Sich-Erheben über einen anderen, in der Reaktion auf einen Lachanlass verständige sich eine Gruppe über sich selbst. ${ }^{8}$ Mit dieser misanthropischen Konzeption greift Bergson die antike Vorstellung auf, die Komödie handle von schlechten Menschen, von Tadel, Misslichkeiten und Fehltritten. Die These kennzeichnet ein pessimistisches Weltbzw. Menschenbild, welches aus der historischen Distanz zum Text betrachtet, verstörend wirken mag, markiert sie doch - ohne ein moralisches Urteil vorzunehmen - das Lachen als Vergemeinschaftungsprozess, in der stets ein Schwächerer ausgegrenzt wird. ${ }^{9}$ So sehr dies auch befremden mag, verweist Bergsons negatives Bild jedoch auf eine spezifische Dimension des Lachens: Anders als im Weinen, dem durch kulturelle Prägungen z.B. im Konzept des Mitleids eine humanistische Prägung innewohnt, drehen sich Lachanlass und Lachen oftmals um Konflikte, soziale Spaltungen und gesellschaftliche Hierarchien.

Zudem ist in den Lesarten von Bergsons Essay die Ausrichtung des Lachens immer wieder auf den Lachenden und den Ausgelachten bzw. das Lachobjekt bezogen worden. Demnach sei das Lachen über jemanden - so Bergson - kalt und distanziert. Weniger beachtet wurde hingegen, dass in Bergsons Zugang das Lachen sehr wohl als affektiv beschrieben wird und zwar als Einbindung, die auf die Gruppe der Lachenden abzielt, die den Grund für ihren Humor teilen. Helmuth Plessner hat später diesen Aspekt von Bergson herausgegriffen und ihn positiv gewendet, wenn er davon spricht, dass dem Lachen stets ein verbindendes Element innewohne, dass das Lachen selten auf das Individuum abziele und vielmehr auf die „Zugewandtheit des Lachenden zur Gemeinschaft“ ${ }^{10}$

6 Siehe Beate Müller: Komik und Komiktheorien. In: Ansgar Nünning (Hg.): Metzler-Lexikon Literatur- und Kulturtheorie. Ansätze - Personen - Grundbegriffe. Stuttgart 2001, S. 317-318. 7 Siehe Bergson 1972 (1900), S. 22.

8 Bergson 1972 (1900), S. 130-131; Anneliese Nowak: Die amerikanische Filmfarce. München 1991, S. 15.

9 Diesen Einwand führt Jurzik ins Feld: „Bergsons Theorie hat Teil an der allgemeinen Aggressivität der Gesellschaft, welche sie gegen alle wendet, die von ihren Normen abweichen. Er hat einem verbreiteten Vorurteil seine theoretische Rechtfertigung geliefert.“ Jurzik 1985, S. 38.

10 Plessner 2003 (1945), S. 332. 
Auch die Forschungsliteratur zur Screwball Comedy greift immer wieder auf Ansätze der Überlegenheitsthese zurück, wenn beschrieben wird, dass z.B. innerhalb der Diegese die weibliche Figur dominant sei, während man als Zuschauer auf die männliche Figur herabblicke. ${ }^{11}$ Ein weiterer Aspekt der Überlegenheitsthese skizziert, dass viele Screwball Comedies das Paar als Teil einer privilegierten, sozialen Gruppe charakterisiere. Die Komödien adressierten zum Teil ein Überlegenheitsgefühl beim Zuschauer gegenüber einer dekadenten, verschwenderischen Upperclass. ${ }^{12}$ In PHILADELPHIA STORY sei andererseits in bester Shakespeare'scher Tradition des Sommernachtstraums - wie Cavell beschreibt das kurzfristige Aushebeln der sozialen Barrieren im Fest und Rausch angesprochen. ${ }^{13}$ Man kann die These zur Überlegenheit mit Cavells Zugriff zusammendenken, nämlich indem man die Hierarchien und Standesdünkel als Teil einer reflexiven Auseinandersetzung der Filme fasst, welche dynamisch ist und die mal in die eine Richtung (als abwertendes Lachen-Über) und mal in die andere Richtung (als Aushebeln von bestimmten Ordnungen) sich bewegt.

2) Die zweite These zu Komik, Witz, Komödie und anderen Lachanlässen betont, auf welche Weise durch das heitere Ereignis eine Entlastung hervorgerufen wird. ${ }^{14}$ Das Lachen wird als eine Art Handlungsprinzip gedeutet, das sich $\mathrm{zu}$ einem emotionalen Ventil der Erleichterung für unterdrückte, angestaute Konflikte entwickelt, an dem sich gesellschaftliche Problematiken aufzeigen. ${ }^{15}$ Bezogen auf die Screwball Comedy wird neben den Themen wie Sexualmoral oder die Berufstätigkeit der Frau auch nach Funktionen des Lachens im Hinblick auf eine Darstellung von Sozialität gefragt: Auf welche Weise die

11 Vgl. Gehring 1986, S. 21.

12 So sieht etwa Steve Vineberg in den Komödien das Genießen einer Exklusivität, die Lust am Besonderen, Außergewöhnlichen, also einen aus seiner Sicht positiven Aristokratismus am Werk, der gerade der These zur Überwindung der Klassenschranken entgegengesetzt ist, siehe Vineberg 2005, S. 5.

13 Cavell 1981, S. 143-144.

14 Zum Begriff der Entlastung siehe Plessner 2003 (1945), S. 297-300.

15 Ursprünglicher Vertreter dieser These ist Freud zum Energieüberschuss in Witz und Komik. So beschreibt Jurzik dies im Zusammenhang mit dem Phänomen des Witzes: „Die Freudsche Formel für den Arbeitsvorgang des Witzes lautet: Vorbewußte Gedanken unterliegen einer blitzschnellen Bearbeitung durch das Unbewußte und werden dann von der bewußten Wahrnehmung erfaßt. Die verschiedenen Techniken des Witzes - Denkfehler, Verschiebung, Verdichtung, Anspielung - haben eine frappierende Ähnlichkeit mit der Technik des Traumes [. . . Die Witzform ist die Bedingung, damit ein obszöner Inhalt in feiner Gesellschaft geäußert werden darf. Sie lenkt die Aufmerksamkeit auf das, was es zu verhüllen galt. Der Witzemacher leistet eine Arbeit, die die Verdrängung, zumindest für einen Moment, außer Kraft setzt.“ Jurzik 1985, S. 47; vgl. Nowak 1991, S. 18. 
Filme in der Zeit der wirtschaftlichen Depression über ihren großen Unterhaltungswert utopische Phantasien schufen. ${ }^{16}$ Zudem wird erörtert, wie in den Komödien gesellschaftliche Rollen und sozialer Status subversiv verhandeln und dadurch Missstände kritisiert werden konnten, ${ }^{17}$ wie sie Fragen $\mathrm{zu}$ Geschlechterrollen und zur weiblichen Emanzipation aufwürfen. ${ }^{18}$

Vertreter der dritten These zur Inkongruenz ${ }^{19}$ betonen, dass es die Unvereinbarkeit bestimmter Polaritäten ist, die zum Lachen anregen. In Anlehnung an klassische Theorien sieht Andreas Furler drei Grundmerkmale der Komödie als ausschlaggebend: „1. Unvereinbarkeit trifft zusammen. 2. Der Zusammenprall verursacht kein Leid, sondern negiert es. 3. Der Zusammenprall ereignet sich überraschend und schnell.“20 Mit diesen drei Merkmalen ist einerseits eine Wahrnehmungsebene angesprochen: das Unvereinbare und die Zeitform des Schnellen. $\mathrm{Zu}$ dem Aspekt des Gefühlsmäßigen als Inkongruität schreibt Noël Carroll:

\footnotetext{
Stated schematically, the incongruity theory of humor says that comic amusement is an emotional state [...]. The perception of incongruity in an event or situation amuses us, which in turn causes the risible sensations - laughter for example - that we feel in response to humor. ${ }^{21}$
}

Dass der „Zusammenprall“ von Unvereinbarem eine gefühlsmäßige Seite hat, die mit dem heiteren Vergnügen zusammenhängt, ist leicht nachzuvollziehen. Doch nicht klar beschrieben ist, wie die Inkongruität des Komischen auf phänomenaler Ebene im Detail zustande kommt. Diese Dimension des Lachens und des komischen Lachanlasses hat Helmuth Plessner u.a. als Mehrsinnigkeit und Ambivalenz beschrieben. ${ }^{22}$

16 Siehe Cavell 1981.

17 Steve Vineberg 2005; Neale und Krutnik 1990; Christopher Beach: Class, Language, and American Film Comedy. Cambridge/New York 2002; Thomas Schatz 1981.

18 Heather Gilmour: Different, Except in a Different Way. Marriage, Divorce, and Gender in the Hollywood Comedy of Remarriage. In: Journal of Film and Video 50 (1998), H. 2, S. 26-39; Jane M. Greene: The Road to Reno. „The Awful Truth“ and the Hollywood Comedy of Remarriage. In: Film History 13 (2001), H. 4, S. 337-358; Petra Coudenhove-Kalergi: Screwball Queens. München 2004.

19 Vgl. Karnick 1995, S. 128; Andreas Furler: Der Guignol in der Zwangsjacke. In: Verband der Studierenden an der Universität Zürich (Hg.): Feste und Feiern. Hollywood-Komödien der 30er und 40er Jahre. Zürich 1990, S. 127-143, hier: S. 132.

20 Furler 1990, S. 132.

21 Carroll 1991, S. 27.

22 Plessner 2003 (1945), S. 328. 
Am reinsten zeigt sich die befreiende Wirkung der Überkreuzung des abdrängenden und des anziehenden Charakters in Situationen der Komik und des Witzes, in denen sich der Antagonismus zwischen anschaulicher Eindeutigkeit und sinnhafter Mehrdeutigkeit, zwischen Sinn und Sinn entfaltet. ${ }^{23}$

Mit Plessners Beschreibung von Mehrsinnigkeit ist nicht einfach eine intellektuelle Doppeldeutigkeit wie im Witz gemeint, sondern eine Situation, die den Körper in ein ständiges Hin und Her zwischen Bindung und Distanz bringt. ${ }^{24} \mathrm{Ob}$ im Kitzel, Spiel, Witz, in der Freude oder Verlegenheit, Plessner fasst die Arten des Lachens allesamt als an dynamische Ausdrucksformen gebunden, in denen der Lachende aufgrund der Paradoxität des Lachanlasses in ständiger Spannung gehalten wird. ${ }^{25}$

Wenn in Bezug auf die Screwball Comedy von Inkongruenz die Rede ist, dann wird damit vor allem die Widersinnigkeit auf narrativer Ebene beschrieben: in Form von Slapstickhandlungen, Gegensätzlichkeit der Figuren oder NonsensElementen. So wird etwa die Polarität des Paares innerhalb der Diegese angeführt, die mit dem Konflikt zweier völlig unterschiedlicher Charaktere einhergehe. ${ }^{26}$ In der Forschungsliteratur wird das Komische jedoch überwiegend auf der Ebene der repräsentierten Handlungen und Narrationen verstanden. ${ }^{27}$

Die drei Begriffe Überlegenheit, Entlastung und Inkongruenz lassen sich im Hinblick auf die Screwball Comedy weniger als konkurrierende Theoreme auffassen, sondern als Teilbereiche eines komödischen Modus. Mit drei ausgewählten Positionen (Quintilian, Bergson und Langer) soll nun vor allem die zeitliche Form der Komödie theoretisch im Fokus stehen.

\section{Die verborgenen Takte der Rede}

Die erste Position ist nicht im Bereich der Komödientheorien verwurzelt, sondern in der Rhetorik. Quintilian spricht die Nähe von affektiven Körperbewegungen und dramatischen Formen an, wenn er nach den Wirkungsweisen expressiver Phänomenen fragt. In der Untersuchung des Rhethors interessiert ihn vor allem die Geste:

23 Plessner 2003 (1945), S. 329.

24 Plessner 2003 (1945), S. 329.

25 Plessner 2003 (1945), S. 277-332.

26 Karnick 1995; Willett 1996, S. 145.

27 Karnick (1995) etwa bezieht sich dabei auf Bergson, Schopenhauer und Kant. Sie versteht incongruity als Brüche in der Narration, S. 128; vgl. King 2002. 
Oder sind nicht sie es (die Bewegungen der Hände, Anmerkung sg), mit denen wir fordern, versprechen, rufen, entlassen, drohen, bitten, verwünschen, fürchten, fragen, verneinen? Mit denen wir Freude, Trauer, Zweifel, Gewißheit, Reue, Maß, Menge, Zahl und Zeit anzeigen ${ }^{28}$

Nicht der Ausdruck subjektiver Gefühle steht im Zentrum, sondern die Geste in ihrer rhetorischen Wirkung. Dabei sei die Tempo- und Zeitgestaltung - die Orchestrierung von Rede und Geste - in hohem Maße dafür entscheidend, dass sich ein Sinn sowie ein überzeugendes weil kongruentes Bild beim Sprechen herstelle. Stimme und Geste müssten synchron aufeinander abgestimmt sein bzw. sich ersetzen oder ergänzen, in keinem Fall aber dürften sie rhythmisch auseinanderfallen. ${ }^{29}$ Obgleich vorher eingeübt sei die gestische Aktivität der Redners so auszuführen, dass sie einen natürlichen und spontanen Eindruck erwecke:

Es gibt gewisse verborgene Takte der Rede und gleichsam gewisse Rhythmen, bei denen die meisten eine Geste machen, wie z.B. Novum crimen, eine Bewegung, C. Caesar, die zweite, et ante hanc diem, die dritte, non auditum, die vierte; dann propinquus meus, und ad te, und Quintus Tubero, und detulit [...]. Von da geht auch jener Fehler aus, daß junge Leute, wenn sie schreiben, die Gesten vorher in Gedanken abmessend so zurechtlegen, wie die Hand absetzen soll. [.. .] Besser ist es, da es in jeder Rede gewisse Glieder gibt, bei denen man, wenn nötig Atem schöpfen kann, diesen entsprechend die Gesten zu verteilen, wie z.B. Novum crimen, C. Caesar, an sich einen gewissen Abschluß hat, weil eine Konjunktion folgt; weiter ist et hanc diem non auditum richtig abgesetzt. Danach muß sich die Hand richten, sofern es sich um ruhige Gebärden des Anfangs handelt. Aber wenn die Hitze sie erregter macht, werden auch die Gesten zugleich mit der Raschheit der Rede häufiger. An den einen Stellen wird ein schneller, an den anderen ein gemessener Vortrag am Platze sein. Mit jenem durchlaufen wir, häufen wir an, eilen wir; mit diesem beharren wir, hämmern wir ein, prägen wir ein. Mehr Pathos hat das Langsamere; so war auch Roscius rascher, Äsopus gewichtiger, weil jener Komödien, dieser Tragödien spielte. ${ }^{30}$

Raschheit und Langsamkeit sind für Quintilian Bewegungsqualitäten (,gewisse verborgene Takte der Rede“, s.o.), die eine Affinität zu anderen Wahrnehmungseigenschaften: das Langsame ist etwa mit Schwere verbunden, (beharren, einhämmern, einprägen). Das Rasche hingegen ist an der Beiläufigkeit orientiert, mit dem wir durchlaufen, anhäufen und eilen. Die sechs Verben entwerfen ein Szenario, welches er durch sinnliche Qualitäten charakterisiert. Mit der Langsamkeit kommen das Prägen von Münzen, die Festigkeit einer Masse,

28 Quintilian in Bühler 1933, S. 229.

29 Quintilian in Bühler 1933, S. 231.

30 Quintilian in Bühler 1933, S. 231-232. 
eine fixierende Kraft in den Sinn. Das Rasche hingegen vervielfältigt, ist flüchtig, momenthaft. In beiden Fällen beschreibt Quintilian, wie die Geste, die der Rhetor intentional einsetzt, sich in der Wahrnehmung realisiert. Die in der Rede eingesetzten Bewegungen der Hände vergleicht er mit Gangarten in den beiden Dramenformen: Das Langsame sei pathetischer, das Gewichtige in der Tragödie vorherrschend, das Rasche bedinge die Leichtigkeit und Wechselhaftigkeit in der Komödie. Diese Idee sinnlicher Bewegungsqualitäten, welche die affektive Wahrnehmung $\mathrm{zu}$ modulieren scheint, entspricht meiner Konzeption der Tempi der Bewegung, wie ich sie in den Kapiteln 3, 4 und 5 entwickelt habe. Dass die Komödie sich durch schnellere, wechselhafte Bewegungen auszeichnet und dass die Bewegungsqualitäten affin sind zur Gewichtswahrnehmung, lässt sich somit auch durch die Position Henri Bergsons bestätigen.

\section{Distanz und Gewichtlosigkeit des Komischen}

Wie andere Positionen der Humor-, Komik- und Lachforschung betont haben, kann Bergson so gelesen werden, als ob er das Lachen rein als kognitive Operation verstehe. Denn er sagt explizit, es sei gerade durch eine Teilnahmslosigkeit des Beobachters gekennzeichnet. Die „Anästhesie des Herzens“31 im Lachen entstehe in erster Linie durch einen intellektuellen Akt. ${ }^{32}$ Doch bereits auf der Ebene der Wahrnehmung sieht er die Unterschiede zwischen komischer Erheiterung und ernsthafter Involvierung:

Sie werden sehen, wie die gewichtslosesten Dinge wie unter der Berührung eines Zauberstabs gewichtig werden, wie alles sich düster färbt. Stellen sie sich nun abseits, betrachten Sie das Leben als unbeteiligter Zuschauer - und manches Drama verwandelt sich in eine Komödie. ${ }^{33}$

Ganz ähnlich wie bei Quintilian - „Mehr Pathos hat das Langsamere“ (s.o.) - hebt Bergson den sinnlichen Eindruck des Gewichts hervor, um einen Gemütszustand mit den Begriffen „Drama“ und „Komödie“ zu charakterisieren. Hinzu kommt, dass sich mit der Gewichtigkeit eine bestimmte Zeitform verbindet: Damit eine Gefühlsorchestrierung im Theater vom Schauspieler aus stattfinde, müsse das „allmähliche Durchdringen“34 des Schauspielers durch die Emotion für die

31 Bergson 1972 (1900), S. 13.

32 Die Frage, ob das Komische an das Bewusstsein gebunden ist, entzweit die Komik-, Humor- und Witztheorien. Vgl. Nowak 1991, S. 18.

33 Bergson 1972 (1900), S. 13.

34 Bergson 1972 (1900), S. 97. 
Zuschauer miterlebbar sein. Die ganze Komposition, alle Bewegungen müssten sich kohärent verbinden, müssten „vibrieren“, so dass die Emotion auch „,ansteckend“ sei. ${ }^{35}$ Die langsame, allmähliche Entfaltung und Nähe eines Ereignisses seien dafür entscheidend. Umgekehrt wird das Komische von Bergson mit Bewegungscharakteristika des Plötzlichen, Abrupten und Überraschenden beschrieben. Dabei unterscheidet er verschiedene Lach-Phänomene, die sich als eine Art Typologie des Komischen, als zeitliche Ausdrucksmuster lesen lassen. So ist die Unterscheidung von Steifheit/Mechanik vs. Lebendigkeit/Elastizität als Grundkomponente des Komischen immer an eine sinnliche Bewegungsdimension gebunden. ${ }^{36}$ Ein weiterer Typus ist der Springteufel, dessen Komik darin besteht, dass er immer wieder in einen Kasten gedrückt wird um nach unregelmäßigen Abständen plötzlich und heftig hervorzuschießen. ${ }^{37}$ Das Prinzip der Wiederholung sowie die darin enthaltenen Gegensätze (unterdrückende und sich befreiende Kraft) sind dafür zentral. Ein weiterer Typus ist der Hampelmann, der im Wesentlichen eine Marionette ist. ${ }^{38}$ Seine Glieder werden bewegt; das heißt, man sieht wie eine Bewegung nicht aus sich heraus geschieht, sondern von einem anderen gesteuert wird. Der Schneeball meint schließlich das Prinzip der Verkettung: Unzusammenhängende Ereignisse werden so aufeinander bezogen, dass sie wie eine rollende Bewegung, ein sich kontinuierlich wachsendes Gebilde wahrgenommen werden. ${ }^{39}$

Der Lachanlass sei laut Bergson immer dann gegeben, wenn sich nicht alle Elemente oder Ausdrucksebenen (z.B. einer Geste) zueinander harmonisch verhalten, sich widersprechen oder Inkongruenzen bzw. Dissonanzen ausbilden. Obgleich er davon spricht, dass das Lachen ein Zustand sei, in dem die Emotion aussetze, zeichnet er doch für den Lachanlass verschiedene Wahrnehmungsszenarien nach, die in erster Linie verkörpert bzw. sinnlich-anschaulich sind. Als sinnliche Anschauungsform betrachtet auch Susanne K. Langer den Rhythmus der Komödie, worin das Lachen gewissermaßen in die dramatische Form eingewoben ist.

35 „So werden wir schließlich selbst von der entsprechenden Gefühlsregung durchtränkt. Man kann auch sagen, eine Emotion sei dramatisch und ansteckend, wenn mit dem Grundton zugleich alle Obertöne angeschlagen werden. Weil der Schauspieler voll und ganz vibriert, kann auch das Publikum vibrieren. Damit uns eine Emotion kühl lasse und infolgedessen komisch wirke, muss etwas Steifes in ihr stecken, das verhindert, dass sie sich mit dem übrigen Teil der Seele verbindet." Bergson 1972 (1900), S. 97-98.

36 Bergson 1972 (1900), S. 32-48.

37 Bergson 1972 (1900), S. 52-53.

38 Bergson 1972 (1900), S. 57-58.

39 Bergson 1972 (1900), S. 58-59. 


\section{Rhythmus und Komödie}

Anders als Bergson versteht Langer das Lachen in erster Linie als gefühlsgebunden. Die Unterscheidung der zwei Dramenformen richtet sie an der gegensätzlichen Zeitgestaltung aus: Die Komödie sei unvorhersehbar und ,episodisch'; sie stelle ein Gleichgewicht sichtbarer Lebendigkeit dar, welche zwar auf das Allgemeine (das Gesellschaftliche) bezogen sei, sich aber in skizzenhafter Gestalt am einzelnen Körper zeige; die Tragödie hingegen beschreibt Langer als ,Erfüllung‘, die eine geschlossene und zielgerichtete Form aufweise. ${ }^{40}$ Den etablierten Gegensätzen vom Gesellschaftlichen der Komödie und dem Individuellen der Tragödie fügt sie eine Zeitform hinzu, welche sie affektpoetisch aber auch in Analogie zu biologischer Animation begründet. Damit wendet sich Langer gegen Ansätze, die von einem sich ähnelnden Aufbau der zwei Dramenformen ausgehen. ${ }^{41}$ Ihre Argumentation liest sich einerseits als Perspektive auf Poetik und Ästhetik, andererseits als lebensphilosophische Auseinandersetzung mit biologischem Einschlag. Die dramatischen Formen verbindet sie mit der Fortpflanzung und dem Lebenszyklus von Lebewesen (Einzellern und höheren Lebewesen). ${ }^{42}$ Die beiden Dramenformen zeichnen sich nach Langer durch bestimmte Grundgefühle aus: Die Tragödie sei an das Erleben einer Endgültigkeit und ihrer entsprechenden Zeitform des Fortschreitens gebunden. ${ }^{43}$ Das Empfinden in der Komödie präge sich hingegen als das Erleben von Vitalität ${ }^{44}$, als das „reine Lebensgefühl“45, als lustvolles Vergnügen und Glück aus. ${ }^{46}$ Langer sieht dabei die Heiterkeit beim Theaterzuschauer mit der künstlerischen Form verflochten: „In einem guten Stück ist das Lachen ein poetisches Element. “47 Darüber negiert sie die Auffassung, dass

40 Langer 1975, S. 137.

41 Langer 1975, S. 128.

42 Das Gefühl in der Komödie sieht sie in Verbindung mit dem sich unendlich vervielfältigenden Einzeller, der sich in andere Lebewesen transformiert; die Tragödie sei hingegen an der Endlichkeit höherer Lebewesen ausgerichtet. Langer 1975, S. 134-135.

43 „Das Leben ist eine Reise, an deren Ende der Tod wartet“. Dieser Gedanke stehe im Zentrum der Tragödie „als Abbild des Verhängnisses“ Langer 1975, S. 136.

44 Langer 1975, S. 150.

45 Das Gefühl der Komödie sei ähnlich zu dem, wenn Menschen zusammenkommen, „in Frühlingsfeiern, Triumphzügen, Geburtstagen, Hochzeiten oder Initiationsriten“, Langer 1975, S. 134.

46 Die Gefahren in der Komödie „sind kein wirkliches Unglück, sondern Unannehmlichkeiten und Verlust des Gesichts“, Langer 1975, S. 156.

47 Langer 1975, S. 147. 
Lachen lediglich eine Reaktion auf die dargestellten Konflikte und Themen sei. Stattdessen hebt sie die rhythmische, poetische und ästhetische Dimension hervor: ${ }^{48}$ „Die wahre Komödie erzeugt im Publikum eine allgemeine Fröhlichkeit, eben weil das Bühnenspiel das Abbild der ,Lebendigkeit‘ bietet, die wahrzunehmen aufregend ist. “49 Die Lebendigkeit in der Komödie bezeichnet Langer mit den Bewegungsqualitäten leicht, fließend, drängend und beschleunigt. ${ }^{50}$ Die Gefühlsregung sei durch eine ganzheitliche Wahrnehmung der Theaterinszenierung bedingt. Es sei nicht das einzelne Gebaren eines Schauspielers, nicht der einzelne Satz, der Erheiterung schafft, sondern das Gefühl der Vitalität erfahre man durch Wechselhaftigkeit, Wiederholung und Rhythmus im szenischen Geschehen. ${ }^{51}$

Die drei skizzierten Positionen betonen zwar sehr unterschiedliche Aspekte: Bergson sieht das Lachen als intellektuell begründet an, verortert es jedoch immer wieder in anschaulichen Bewegungs-Phänomenen; Langer versteht es als gefühlsgebundenes Erleben; Quintilian geht es nicht ums Lachen, sondern um die Bewegungsqualitäten, die sich in Gesten, aber auch in Dramen wiederfinden. Doch die Schnittstellen der drei vorgestellten Positionen sind ebenso offensichtlich: Das Komische wird als bildliche Anschauungsform verstanden. Die Wechsel der Tempi im Vortrag und im Drama sind auf das Publikum wirksam (Quintilian); die Wahrnehmungsform des komischen Ereignisses lenkt die Aufmerksamkeit des Betrachters auf die Äußerlichkeit und das Bewegungsmoment des Geschehens (Bergson); das Lachen ist als poetisches Element ein Teil der Komödie (Langer). Zudem fassen alle drei Positionen den komisch-komödischen Wahrnehmungsmodus als zeitlich strukturiert auf: Quintilian beschreibt die Gestenformen in der Komödie als rasch; Bergson versteht die Komik als an sinnliche Muster des Schnellen, der Wiederholung, des Abrupten, des Widersinngen und der Distanz gebunden. Langer fasst die Komödie als leicht, schnell und durch Wiederholung geprägt auf, die den Gefühlsmodus und das Vitalitätsempfinden ansprechen.

Die drei Positionen beschreiben, auf welche Weise eine spezifische Zeitlichkeit für den komisch-komödischen Modus ausschlaggebend ist. Diese Bewegungs- und Ausdrucksqualitäten lassen sich zusammenfassen als wechselhaft, abrupt-ungewiss, plötzlich, mechanisch, wiederholend, anhäufend, schnell, leicht,

48 Langer 1975, S. 151.

49 Langer 1975, S. 154.

50 Langer 1975, S. 155-156.

51 „Diese Illusion von Leben, das Bühnengeschehen, hat einen Gefühlsrhythmus, der uns nicht durch einzelne aufeinanderfolgende Reize übermittelt wird, sondern eher dadurch, daß wir seine ganze Gestalt erfahren [. . .].“ Langer 1975, S. 155. 
flink, elegant, äußerlich, klein, zart. Im Zusammenhang mit der Screwball Comedy lässt sich aus den letzten Kapiteln resümieren, dass sich dieser komödische Modus stets historisch und kulturell spezifiziert bzw. am einzelnen Film bzw. in seinem Genre im Zusammenhang mit seinen Inszenierungsformen herausbildet.

\title{
6.3 Zur Dynamik des Genres
}

\section{Überlegungen zu Genretheorien}

Warum Filme immer wieder ihre Zuschauer ansprechen, auch nach wiederholtem Sehen, warum eine Komödie jedes Mal erneut erheitert, obwohl die Erzählung längst bekannt ist, diese Fragen lassen sich auch auf einen größeren Zusammenhang beziehen. So schreibt Knut Hickethier im Zusammenhang mit Genrefilmen generell:

\begin{abstract}
Nicht zuletzt hier stellt sich die Frage, warum sich denn immer wieder Millionen von $\mathrm{Zu}$ schauern diese Geschichten ansehen, deren Struktur längst bekannt ist und deren Ablauf sich im Wesentlichen immer gleich gestaltet. Das Interesse kann nicht allein in den Plots liegen, weil der Ausgang fast immer gleich ist. Das Ritual der Genrenutzung und der Genrelektüre zielt also auf tiefer liegende Botschaften. ${ }^{52}$
\end{abstract}

Hickethier benennt das Problem zielgenau: Die „tiefer liegenden Botschaften“ sind Phantome, welche die Genretheorie umtreiben. Zwar ist es Konsens, dass Genre ein Verständigungsbegriff ist, der von Produzenten, Filmkritikern, Zuschauern und anderen Gruppen im Rahmen der Kommunikation über Film genutzt wird. ${ }^{53}$ Die Theorien unterscheiden sich in der Antwort auf die Frage, welche Eigenschaften die Filme eines Genres verbinden. In diesem Zusammenhang verzweigen sich die Perspektiven auf das, was sich in den Filmen wiederkehrend auffinden lässt: von produktionstechnischen oder industriellen Standards über narrative Konventionen und Regelpoetiken, die meist auf repräsentierten Inhalten basieren oder sich auf soziale und kulturelle Sachverhalte beziehen. ${ }^{54}$ Ist von einer Entwicklung eines Genres die Rede, dann gehen die meisten Zugänge von einer Idee aus, die mit Schema und Variation

52 Knut Hickethier: Genretheorie und Genreanalyse. In: Jürgen Felix (Hg.): Moderne Film Theorie. Mainz 2007, S. 62-96, hier: S. 86.

53 Hickethier 2007, S. 63. Neale und Krutnik 1990.

54 Vgl. Kappelhoff 2016, S. 86-96. 
vergleichbar ist (bzw. entsprechenden Begriffen), sie stimmen darin überein, dass in einem Genre gewisse Elemente (Figuren, Thema, Handlungsabfolgen, Motive) sich ständig wiederholten, während andere Elemente variiert würden. ${ }^{55}$ Viele Genretheorien sehen unter anderem den Vorgang der Konventionalisierung ${ }^{56}$ oder der Sehgewohnheit ${ }^{57}$ als zentral für die Bildung eines Genres an. Zugänge zu ,Genre als Prozess“ können grob in zwei Richtungen eingeteilt werden: ${ }^{58}$ Zum einen wird die Transformation von Genres betont, das heißt, die Variation wird fokussiert. Zum anderen gibt es Konzepte, welche die Formulae, das fixe Schema, die prototypischen Szenen in den Mittelpunkt stellen, um die Stabilität von Genres über einen gewissen Zeitraum aufzuzeigen. ${ }^{59}$ Die These, Genres wandelten sich historisch, wurde immer wieder prominent verhandelt. ${ }^{60}$ Einer rein chronologischen Vorstellung von Zeit widerspricht z.B. der Zugang Stanley Cavells, der mit dem Begriff der Familienähnlichkeit ein sehr dynamisches Bild von Genre zeichnet und die einzelnen Filme als Denkformationen ansieht. Cavells Modell wird zunächst dargestellt, um es dann auf filmwissenschaftliche Zugänge zum Genre zu beziehen, die den Erfahrungsmodus für Genres zentral setzen. ${ }^{61}$

\section{Familienähnlichkeiten}

Stanley Cavell greift mit seinem Konzept der Familienähnlichkeit auf die naheliegende etymologische Verbindung von Genre und Genus zurück; seine Auffassung liegt gerade nicht im Fixieren systematischer Ebenen begründet, im Abstrahieren von Mustern, die viele Genretheorien umtreibt. Ich werde sein Konzept, das er am Filmzyklus der Remarriage Comedies entwickelt hat, darstellen und es auf das umfassendere Genre der Screwball Comedies beziehen.

55 Vgl. Karnick 1995.

56 Vgl. Edward Buscombe: The Idea of Genre in the American Cinema. Screen 11 (1970), H. 2, S. 33-45; Steve Neale: Genre and Hollywood. London 2000, S. 12-13.

57 Neale 2000, S. 17-19.

$58 \mathrm{Zu}$,Genre als Prozess‘ siehe Steve Neale: Questions of Genre. In: Barry Keith Grant (Hg.): Film Genre Reader II. Austin 1995; Rick Altman: Film/Genre. Basingstoke/London 1999, S. 159-183, hier: S. 49-86.

59 Siehe Jenkins und Karnick 1995, S. 2.

60 Im Sinne eines „Genre as process“, Neale 1995, S. 170. Vgl. Altman 1999, S. 21.

61 Siehe Kappelhoff 2016, S. 96-97. 
Stanley Cavell schreibt in seinem Buch Pursuits of Happiness, dass jeder Film, der einem Genre angehört, erst im gemeinsamen Bezug zu anderen Filmen zu verstehen sei. Für Cavell beginnt ein Genre mit einem ersten Film; mit diesem Film ist das Genre zu diesem Zeitpunkt im Prinzip bereits vollständig ausgebildet („full-blown“). ${ }^{62}$ Im Falle der Remarriage Comedy (ein Filmzyklus innerhalb der Screwball Comedy), nennt er IT HAPPENED ONE NIGHT. Unter Vollständigkeit versteht er, dass es dem Genre zu diesem Zeitpunkt an nichts mangele. ${ }^{63}$ Das Genre sei bereits von Beginn an voll entwickelt, jedes Muster, jede Szene, jedes kompositorische Element, das der Film ausbildet, ist gleichzeitig sein Genre-Merkmal. Alle genrespezifischen Eigenschaften sind zu diesem Zeitpunkt komplett. Dann mit dem zweiten Genrefilm, der etwas später herauskommt, ändere sich das gesamte Genre. Wiederum fließen alle Elemente des zweiten Films als Genremerkmale in das Genre ein. Cavells Ansicht ist, dass alle Szenen eines Films wichtig sind und nicht ein Film typischer oder untypischer sein kann. Und so geht es Film für Film weiter. Diese Auffassung ist konstruktivistisch und orientiert sich an der Positivität der Filme, dass sie zum Zeitpunkt ihres Auftauchens einen bestimmten Entwicklungsstand des Genres beschreiben. Dabei gibt es nun nicht ein Merkmal, das alle Filme teilten:

The idea is that the members of a genre share the inheritance of certain conditions, procedures and subjects and goals of compositions, [...]. There is, on this picture, nothing one is tempted to call the features of a genre which all its members have in common. ${ }^{64}$

Alle Szenen, alle Elemente, alle Kompositionsmuster in einem Film sind somit zentral für das Genre, dass in seiner zeitlichen Entfaltung so etwas wie Familienähnlichkeiten ausbildet. Mit dem Konzept bezieht Cavell sich auf Wittgenstein, der mit dem Begriff Spiele in den Blick nimmt:

Betrachte z.B. einmal die Vorgänge, die wir „Spiele” nennen. Ich meine Brettspiele, Kartenspiele, Ballspiele, Kampfspiele, usw. Was ist allen diesen gemeinsam? - [. . ] wenn du sie anschaust, wirst du zwar nicht etwas sehen, was allen gemeinsam wäre, aber du wirst Ähnlichkeiten, Verwandtschaften, sehen, und zwar eine ganze Reihe. Wie gesagt: denk nicht, sondern schau! - Schau z.B. die Brettspiele an, mit ihren mannigfachen Verwandtschaften. Nun geh zu den Kartenspielen über: hier findest du viele Entsprechungen mit jener ersten Klasse, aber viele gemeinsame Züge verschwinden, andere treten auf. [...]

62 Cavell 1981, S. 28.

63 Diese künstliche Setzung eines ersten Films dient dazu, bewegliche Schnitte durch die Geschichte zu machen, welche es ermöglichen, eine Genredynamik in ihrer Bewegung nachzuzeichnen. Anschließend wäre aber ein solcher rekonstruierter Zyklus wieder im Bezug zu weiteren Zyklen und Genres zu situieren, zu deren Vorläufern, Ahnen und Verwandtschaften. 64 Cavell 1981, S. 28. 
Und so können wir durch die vielen, vielen anderen Gruppen von Spielen gehen. Ähnlichkeiten auftauchen und verschwinden sehen. Und das Ergebnis dieser Betrachtung lautet nun: Wir sehen ein kompliziertes Netz von Ähnlichkeiten, die einander übergreifen und kreuzen. Ähnlichkeiten im Großen und Kleinen. ${ }^{65}$ [. . . I Ich kann diese Ähnlichkeiten nicht besser charakterisieren, als durch das Wort „Familienähnlichkeiten“; denn so übergreifen und kreuzen sich die verschiedenen Ähnlichkeiten, die zwischen den Gliedern einer Familie bestehen: Wuchs, Gesichtszüge, Augenfarbe, Gang, Temperament, etc. etc. - Und ich werde sagen: die ,Spiele‘ bilden eine Familie. ${ }^{66}$

Wittgenstein skizziert mit seinem Konzept, das er für Sprache geltend macht, „eine Familie von Bedeutungen“ ${ }^{67}$ Dabei zielt er auf Kontextualität und Erfahrungszusammenhänge von Sprache $\mathrm{ab}^{68}$ und ersetzt die Vorstellung einer fixierten Wortbedeutung durch einen gelebten Sprachgebrauch. ${ }^{69}$

Cavell überträgt das Konzept von der Sprache auf Filmgenres und versteht dabei eine Gruppe von Filmen nicht durch eine Sammlung fixer, klassifizierbarer Eigenschaften verbunden. Vielmehr beschreibt er, wie sich die Filme aufeinander beziehen und Verbindungen herstellen als gemeinsame Reflexion über ein bestimmtes Thema. Genretransformation ist demnach als Prozess ins Offene $\mathrm{zu}$ begreifen. Im Prinzip reflektierten die Filme einander: „They are what they are in view of one another.“70

Die Filme sind erst im Zusammenhang, im dynamischen Bezug zueinander bestimmbar. Cavell sagt, dass die Filme in ihrem Entfalten einen bestimmten Mythos etablierten, den sie bearbeiteten, ausdrückten bzw. reflektierten. ${ }^{71}$ Die Filme bilden Relationen zueinander aus, spiegeln einander, beziehen sich aufeinander. Dies ist nun weniger als atemporale Intertextualität zu verstehen denn als Fortschreiten und Aufnehmen von Topoi und Denkformen, die je spezifisch in den historischen Konstellationen situiert sind. Cavell sieht innerhalb seines Konzepts

65 Wittgenstein 1984a, § 66.

66 Wittgenstein 1984a, $\S 66$ und 67.

67 Wittgenstein 1984a, § 77.

68 Wittgenstein 1984a, § 43.

69 Zur Familienähnlichkeit in der Sprache schreiben Gebauer und Stuhldreher: „Mit diesem Konzept gibt er den Gedanken auf, dass Begriffe durch eine klar begrenzte Menge genau bestimmbarer Merkmale definiert werden können. An die Stelle von Wesensmerkmalen setzt Wittgenstein die Vorstellung von Ähnlichkeiten, die zwischen verschiedenen Erscheinungsformen des gleichen Sprachspiels bestehen." Gunther Gebauer und Anna Stuhldreher: Wittgenstein. Das Sprachspiel der Emotionen. In: Hilge Landweer und Ursula Renz (Hg.): Klassische Emotionstheorien. Von Platon bis Wittgenstein. Berlin/New York 2008, S. 615-634, hier: S. 681.

70 Cavell 1981, S. 29.

71 Cavell 1981, S. 31. 
der Familienähnlichkeit die Filme als eigenständige Individualitäten an. ${ }^{72}$ Erst wenn eine direkte Nachahmung von vorhergehenden Filmen der Fall ist, sei das Genre meist am Ableben, es habe dann die gemeinsamen Perspektiven zur Genüge ausgeschöpft und neue Filme würden sich nicht mehr reflexiv auf frühere Filme beziehen. Wenn ein Genre sich erschöpft habe, trete die Sättigung seiner Expressivität ein. ${ }^{73}$

Damit ist eine extrem dynamische Perspektive umrissen, welche den Prozess von Filmgeschichte in den Blick nimmt. Mit Cavell verstehe ich Genre demnach als dynamische Reflexionsform zu bestimmten Themen. Die Themen selbst speisen sich aus den Diegesen, Fiktionalisierungen, Figuren, sind aber meist auf gesellschaftliche, soziale Fragen bezogen, die dadurch reflektiert werden z.B. die große Kluft zwischen Arm und Reich während der Großen Depression. Doch das Reflektieren über die Themen setzt niemals an der Wiederholbarkeit von Mustern an, das Denken der Filme ist dabei stets an die konkreten Formen von Inszenierung und Expressivität gebunden, welche jeweils spezifisch ausgebildet werden und mit dem ein Film je Eigenes schafft (dem Genre neu hinzufügt) oder mit dem er eine Perspektive auf vergangene Ausdrucksformen von Filmen richtet. Freilich ist die Reflexion an die Tatsache gebunden, dass die Filme eines Genres von Zuschauern verkörpert werden, von Zuschauerinnen welche vergangene Filme selbst erfahren haben. Die Familienähnlichkeiten fasse ich daher nicht einfach als strukturelle Elemente der Verbindung, vielmehr verstehe ich sie als performative Elemente, die Erinnerungen an frühere Filmerfahrungen, an Gefühle, gedankliche Assoziation in ihren Zuschauerinnen aufrufen. In eben diesem Sinn kann man auch den historischen und gesellschaftlichen Bezug der Komödien zu ihrer Zeit bestimmen. Sie sind begreifbar als mediale Formen der Phantasietätigkeit sowie der Gefühlskultivierung, wodurch bestimmte Themen im Austausch der Filme mit ihren Zuschauern bearbeitet und ergründet werden. ${ }^{74}$ Die Filme sind selbst an einem bestimmten Gespräch über das Gesellschaftliche beteiligt. Thematische Begriffe, welche in den Diegesen der Filme aufscheinen, wie z.B. Ehe, Geschlechterrollen oder die wirtschaftliche Existenz sind nicht

72 Vgl. Maria-Sibylla Lotter: Das Abenteuer der Alltäglichkeit. In: Stanley Cavell: Cities of Words. Ein moralisches Register in Philosophie, Film und Literatur. Zürich 2010 (Legierungen. Bd. 7), S. 7-24, hier: S. 17.

73 ,[S]aturation of expressiveness“, Cavell 1981, S. 33.

74 Cavell macht keinen Unterschied zwischen philosophischen Texten und populären Filmen, beide sind für ihn Reflexionsformen. Siehe Lotter 2010, S. 14-15. Zum Denken der Filme siehe Kappelhoff 2018, S. 9-70, 163-184. 
einfach dargestellte Hintergründe für eine Erzählung der Screwball Comedies, sondern man kann an den sich entfaltenden Genreformationen der Filme selbst ein Nachdenken, ein Abwägen, Argumentieren oder Verwerfen im Bezug zu solchen Themenbereichen erkennen. ${ }^{75}$ Dies realisiert sich allein auf der Ebene der filmischen Expressivität, welche im Zuschauererleben ein Denken zu strukturieren vermag, gleichzeitig aber auch radikal eigenständig als ein eigenes Denken der Filme erfahrbar wird. ${ }^{76}$

$\mathrm{Zu}$ der Phantasietätigkeit der Genres gehört ebenso eine gefühlsgestaltende Seite, welche die Basis für das Denken in und mit filmischen Bildern überhaupt erst begründet. Dies lässt sich durch Ansätze stützen, welche die affektiven und körperlichen Erfahrungsdimensionen von Genre betrachten. ${ }^{77}$ Sie entwerfen ein bestimmtes Verhältnis von Film und Zuschauer, das im verkörperten Akt des Filmwahrnehmens begründet ist. Statt ganze Filme klassifikatorisch einem fixen Genre zuzuordnen, hat sich die aktuelle filmwissenschaftliche Forschung (der ästhetisch-philosophischen Linie) darauf fokussiert, den genrespezifischen Erfahrungsmodus in den Mittelpunkt zu rücken. Kappelhoff schreibt:

Statt einzelner Genres sind hier generische Modi eines genrepoetischen Systems als spezifische Expressivitäten und affektgenerierende Modalitäten unterschieden (die sentimentale Traurigkeit des Melodramas, das Lachen der Komödie, der Angstthrill des Horrorgenres, der

75 Rick Altman hat die Art und Weise, wie Filme selbst deutend tätig sein können, mit dem Begriff des symbolischen Gebrauchs besetzt. In einem Western etwa seien nicht die Gegenstände oder Orte entscheidend (Prärie, Waffe, Saloon), sondern ihr „symbolic usage of key images“, wie sie eingebunden sind in eine sinnhafte Figuration: „Actual location matters less for the Western's incessant long shots of the landscape than the way the landscape is used to figure the simultaneous danger and potential that the Western represents." Altman 1999, S. 26.

76 Kappelhoff hat gezeigt, auf welche Weise die Filme eine Spaltung des Zuschauererlebens und -denkens evozieren: die Anordnung der Einstellungen in der Montage strukturiert nicht nur das Denken von Zuschauern, sondern präsentiert eine Eigenständigkeit, mit der Zuschauer immer schon von dieser Wahrnehmung ausgeschlossen sind. Die Erzeugung fiktionaler Welten ist ein ,als ob', ähnelt unserer Welt, assoziert eine uns bekannte Welt und schafft aber gleichzeitig immer eine komplett eigene fiktionalisierte Welt, an der wir wahrnehmend und empfindend, denkend teilnehmen können, in die wir aber niemals eingreifen können. Kappelhoff 2018, S. 57-67.

77 Vgl. Christine Gledhill: Rethinking Genre. In: Christine Gledhill and Linda Williams (Hg.): Reinventing Film Studies. London 2000, S. 221-243; Linda Williams: Film Bodies. Gender, Genre, and Excess. In: Robert Stam und Toby Miller (Hg.): Film and Theory. An Anthology. Malden/Oxford 2000, S. 207-221; Thomas Elsaesser: Zu spät, zu früh. Körper, Zeit und Aktionsraum in der Kinoerfahrung. In: Matthias Brütsch et al. (Hg.): Kinogefühle. Emotionalität und Film. Marburg 2005, S. 415-440; Hermann Kappelhoff 2004a. 
Suspense des Thrillers). Aus meiner Sicht ist damit die moderne Genrepoetik selbst als eine Kombinatorik von Affektmodalitäten beschrieben. Diese hält die Möglichkeit unterschiedlichster affektdramaturgischer Schemata bereit, welche sich wiederum auf die verschiedensten Medientechnologien und poetischen Darstellungstechniken beziehen lassen. ${ }^{78}$

Die Genremuster, welche unterschiedliche Filme miteinander verbinden, basieren so gesehen nicht auf narrativen Standards - eine Wiederkehr von Figurentypen, Erzählmotiven und Handlungsschemata - sondern zeichnen sich in erster Linie durch einen affektiven Erfahrungsmodus aus, der innerhalb eines Genres dominant wird (oder aber in Hybridformationen präsent ist). So schreibt Hermann Kappelhoff:

Die Modi des Genrekinos bezeichneten dann solcherart unterschiedene Formen ästhetischen Genießens. Das Genrekino wäre auf dieser Ebene als ein System unterschiedlicher expressiver Modalitäten zu verstehen, welche die Affektivität der Zuschauer adressieren, um diese in einer gemeinschaftlich geteilten Empfindungswelt zu verorten - in diesen Modulationen wäre das individuelle Empfinden der Zuschauer immer schon in seiner Verwobenheit mit einer kollektiven Gefühlswelt greifbar. ${ }^{79}$

Aus dieser Sicht sind Filmgenres nicht an Regelpoetiken oder Plot-Schemata gebunden, sondern ermöglichen mediatisierte Praktiken, ein „poetisches Machen“80, welches von Zuschauerinnen über ihre Erfahrungen generiert wird; gleichzeitig fungieren diese Praktiken als eine gemeinschaftliche Verständigung, ein Austausch in Form von Erfahrungs- und Reflexionsformen. So schreiben Grotkopp und Kappelhoff:

Genres as realisations of modalities of experience create historically and culturally variable „poetics of affect“. They are aesthetic forms of situating the spectator in a shared space of perceiving, feeling, and thinking: Genres and their instantiations are testimonies to a faculty of imagination that is capable of crossbreeding one's embodied experience with the fantasies of others. ${ }^{81}$

Der Begriff der Affektpoetiken bündelt den jeweiligen Zusammenhang von Denken, Empfinden und Wahrnehmen und zeichnet ihn als einen gemeinschaftlich geteilten Erfahrungsmodus aus. Über die Filmrezeption sind Zuschauerinnen verbunden, sie teilen dieselbe mediatisierte Anordnung von Wahrnehmung und verkörperter Erfahrung. Die Gefühlsmodi, Reflexionsweisen und Formen filmischer Welterzeugung sind dabei auf der einen Seite höchst artifiziell hergestellt, in

78 Kappelhoff 2016, S. 103.

79 Kappelhoff 2016 S. 104.

80 Kappelhoff 2016 S. 104.

81 Kappelhoff und Grotkopp 2012, S. 9. 
ihrer Existenz als Kulturprodukte, auf der anderen Seite realisieren sie sich als solche erst in der körperlichen und phantasierenden Prozessualität einer aktiven Zuschauererfahrung. ${ }^{82}$ Wie sich die Familienähnlichkeiten der Filme sowie deren spezifische Erfahrungsmodalitäten konkret im Genre der Screwball Comedy ausprägen, möchte ich im Folgenden aufzeigen.

\subsection{Szenische Familienähnlichkeiten}

Die folgenden Familienähnlichkeiten sind inszenatorische, affektpoetische und narrative Eigenschaften von Szenen innerhalb des Genres der Screwball Comedy, die ich aus dem Forschungsstand ${ }^{83}$ und eigenen Analyseergebnissen entwickelt habe. Ich nenne sie Kategorien, jedoch sind sie nicht als fixe, sich wiederholende Schemata gedacht, sondern als Teilelemente eines Themas bzw. einer Phantasie, welche die Filme gemeinsam und in Bezug zueinander reflektieren. Aus den Kategorien von Familienähnlichkeiten wie sie von den Filmen inszeniert werden, wird ein Kompositum aus Sinnbezügen ausgebildet, welches sich als das übergreifende Thema fassen lässt. Das bedeutet, dass sie häufig aber nicht in allen Filmen vorkommen. Es kann auch einen Film geben, der nur mit wenigen Filmen (aus dem Genre) Ähnlichkeiten teilt. Einige Filme bearbeiten etwa besonders eine bestimmte Kategorie und lassen andere fallen. Die Kategorien stellen demnach nicht Prototypenszenen dar, sondern sind reflektierende Sinn- oder Themenbezüge; Zudem begreife ich, wie oben ausgeführt, nicht nur die Kategorien als das Genre konstituierend, sondern vielmehr alle Szenen aller beteiligten Filme. Die Kategorien sind jedoch eine Art Essenz des Genre-Charakters, des genrespezifischen, affektpoetischen Erfahrungsmodus, eine grobe Skizze der wichtigsten Eigenschaften. Ich werde sie ich im Folgenden in einzelnen Abschnitten beschreiben.

\section{Vision eines reichen Lebens}

Die Kategorie prägt sich in vier Formen aus, sie handelt von der Vision eines reichen, mondänen und souveränen Lebens oder aber von der einer reichen,

82 Kappelhoff 2018.

83 Besonders habe ich mich in der Entwicklung an Tamar Jeffers McDonalds Kategorien der Romantic Comedy orientiert, vgl. McDonald 2007. Zu den Szenen-Kategorien „meet cute“ und „wrong partner“ siehe auch Steve Neale: The Big Romance or Something Wild? Romantic Comedy Today. Screen 33 (1992), H. 3, S. 284-299. 
dekadenten, verschwenderischen Gesellschaft. Mit den Ausformungen sind unterschiedliche Gefühlsmodi verbunden:

1) Die sehnsuchtsvolle Phantasie eines reichen Lebensstils inszeniert den Wunsch nach unbeschwertem Umgang mit Geld und Gütern: Edle Kleidung, Schmuck und teure Accessoires in glänzendes Licht gesetzt, elegante Appartements, Herrenhäuser und exklusive Tanzlokale sind die Ausstattung und Kulissen vieler Szenen. Die Zeitlichkeit der Inszenierung gleicht dabei z.B. einem andächtigen Staunen, in dem sehr langsam und weichgezeichnet Details von Stoffen, Materialien und Oberflächen hervorgehoben werden. Man sieht etwa Mary Smith (Jean Arthur) in EASY LIVING als modernes Aschenbrödel, wie sie die luxuriöse Hotelsuite durchschreitet. Langsam entfaltet sich in der Montage eine Öffnungsbewegung, das Erschließen verschiedener, immer neuer Räume. Im Wechsel mit der Ausstattung der Zimmer, deren Begehung fast einer Begegnung mit einem Wesen gleicht, das sich von seiner besten Seite zeigt, sieht man das staunende Gesicht Jean Arthurs. Die Räume erscheinen dabei fast personifiziert: Ein Lichtwechsel, ein Türöffnen ähnelt einer einladenden Geste. Kaum zieht Mary sachte an einer Kordel, so öffnet wie von magischer Hand ein breiter Vorhang die Fensterfront, in anmutiger Weise weicht der Stoff zur Seite, wie die Rockschöße einer Frau beim Tanz wippt er hin und her, helles, warmes Licht strömt in den großzügigen Salon. Die extreme Ausstellung von Luxuswaren, teuren Kleidern und anderen Gütern, Elemente aus der Werbeästhetik, Darstellungen des Konsum- und Freizeitverhaltens werden immer wieder in die Screwball Comedies eingebracht. Das hat den Komödien vielfach den Ruf eines eskapistischen Genres eingebracht. ${ }^{84}$ Neben der Orchestrierung eines langsamen Staunens angesichts der Warenfetischisierung kann man eine weitere Zeitgestaltung ausmachen, welche eher bei der Inszenierung immaterieller Statussymbole $\mathrm{zu}$ beobachten ist (Kreuzfahrten, Fliegen, Bahnfahren, Nachtclub-, Hotel- und Restaurantbesuche). Einstellungen unterschiedlicher Freizeitaktivitäten werden rasant hintereinander geschnitten, als rase die Zeit wie in einem Vergnügungspark. Das Paar erscheint dabei stets kurz porträtiert vor dem Hintergrund von Reiseschauplätzen, Pferderennen oder beim Wassersport (z.B. THE AWFUL TRUTH). Beide Zeitformen der Kategorie Vision eines reichen Lebens - das langsame Staunen und das achterbahnartige Vergnügen - reflektieren die Ausstellung von Wohlstand auf eine Weise, welche die Phantasie eines reichen und mondänen Lebens affektpoetologisch entweder als sehnsuchtsvolles Schwelgen, als Versunkenheit oder als kurzweiligen Geschwindigkeitsrausch erscheinen lässt.

84 Seeßlen 1976, S. 80. 
2) Die zweite Ausformung der Kategorie Vision eines reichen Lebens ist als das Gegenteil zu beschreiben. Sie meint eine kritische, persiflierende oder sarkastische Perspektive auf eine reiche Gesellschaftsschicht. Dabei wird die Skurrilität, Verschwendungssucht, das exzentrische Verhalten von Menschen, die im Überfluss leben, aufgezeigt. So wird in THE PALM BEACH STORY eine Millionärin als exaltierte und hysterische Person gezeichnet, die einen Mann zu ihrer Belustigung wie ein Haustier hält. In MY MAN GODFREY wird eine völlig verschwenderische High Society dargestellt, deren einzige Beschäftigung das Verprassen von Geld ist. Darin deuten die Filme eine Vision von Reichtum als Dekadenz. Auch Capras Screwball Filme sind allesamt hier anzusiedeln, so etwa das Verhalten des Vaters der Braut, das in IT HAPPENED ONE NIGHT extrem überspitzt gezeichnet wird. Neben den narrativen Darstellungen sind vor allem Slapstick-Elemente zu nennen: Die sinnlose Zerstörung von Gütern, das Zerbrechen von Gegenständen werden in vielen Screwball Comedies prominent ausgestellt. In BRINGING UP BABY gräbt ein Paar auf der Suche nach einem Knochen einen riesigen, sauber angelegten Garten um. In UNFAITHFULLY YOURS zerstört die Hauptfigur aus Tollpatschigkeit das Mobiliar einer ganzen Wohnung; YOU CAN'T TAKE IT WITH YOU ${ }^{85}$ zeigt, wie Tische, Stühle und Geschirr eines Restaurants in nur wenigen Minuten völlig über den Haufen geworfen werden. In EASY LIVING bricht ein Automatenrestaurant zusammen, Geschirr und Essensreste häufen sich übereinander. Immer wieder wird in Montagesequenzen der Zerstörung von Dingen längere Zeit gewidmet; wie eine Ordnung sich in ein Chaos verwandelt. Die Gefühlsmodi, die damit einhergehen mögen Empörung oder Belustigung über die sinnlose Verschwendung sein. Hier sind es vor allem die unterschiedlichen Bewegungsqualitäten, die erheitern: das Plötzliche des hysterischen Anfalls, die Abruptheit sich zersetzender Räume bei gleichbleibenden Einstellungen und statischer Kamera. Die Unterkategorie ist besonders historisch spezifisch ausgeformt. Man kann davon ausgehen, dass die Zerstörung von Essbarem sowie von teuren Gütern in einer Zeit, in der viele Amerikaner aufgrund der Großen Depression Hunger litten, einen provokativen, aufrührerischen Charakter besaß bzw. für Zuschauerinnen geradezu ethisch fragwürdig war.

3) Die dritte Ausprägung der Vision eines reichen Lebens gilt der Thematisierung ökonomischer Aspekte. Es wird in den Komödien nicht nur von Geld gesprochen, sondern auch mit sprachlichen Äußerungen aus dem Bereich des Ökonomischen operiert, um eheliche Beziehungen $\mathrm{zu}$ metaphorisieren. Dabei können die 
verbalen Markierungen in ihrer Funktion sowohl als Vision eines reichen, unbeschwerten Lebens bzw. einer Dekadenz eintreten. Zudem gibt es audiovisuell-inszenatorische Darstellungsformen, welche im Akt des Sehens ein Beziehungsgefüge zu Ansichten des Tauschs und Handels umwandeln, so etwa im Bildtypus des kommunizierenden Gesichts.

4) Die vierte Ausprägung der Kategorie entwirft aus den Fragen nach Reichtum und Ökonomie eine Perspektive auf soziale Rollen. In vielen Filmen entwickeln sich die Figuren anhand von Oppositionen, welche die Protagonisten verkörpern. Das Paar vereint sich am Ende des Films und verbindet dadurch Dichotomien wie arm und reich (sowie auch großstädtisch und ländlich, proletarisch und adlig, etc.). ${ }^{86}$ Nach diesem Muster sind viele Filme aufgebaut. Dabei kommt das ökonomische Prinzip besonders häufig vor. So das AschenbrödelMotiv: Eine arme Frau gelangt unverhofft in gehobene Kreise oder gibt sich als wohlhabende Dame aus (MIDNIGHT, HANDS ACROSS THE TABLE ${ }^{87}$, THE LADY EVE, EASY LIVING, THE PALM BEACH STORY). ${ }^{88}$ Auch die männliche Hauptfigur aus einfachen Verhältnissen heiratet in die Upperclass ein (PLATINUM BLONDE, MY MAN GODFREY, IT HAPPENED ONE NIGHT oder HOLIDAY). Die Kategorie ist durch und durch von den Filmen Capras geprägt. Durch die Paarbildung werden soziale Gegensätze, die über den Film hinweg aufgebaut werden, überwunden. ${ }^{89}$

\section{Erzieherische Maßnahme}

Diese Kategorie hat sich in vielen Filmen herausgebildet (THE LADY EVE, MY MAN GODFREY) und ist besonders stark durch Frank Capras Filme (z.B. IT HAPPENED ONE NIGHT) geprägt. Die moralische Besserung einer Figur steht dabei im Zentrum, wobei man als Zuschauer lernt, dass es darum geht, das Individualwohl

\footnotetext{
86 Siehe Willett 1996, S. 144.

87 HANDS ACROSS THE TABLE (Mitchell Leisen, USA 1935).

88 Das ganze Genre wird mitunter auf die Formel des Aschenputtel-Märchens enggeführt. Vgl. Sennett 1973, S. 20.

89 So Melling: „Frank Capra's comedies reached out to their audiences and offered reassurance through a dream of social unity. [...] he never made his audiences feel insufficiantly uncomfortable to need to seek revenge on the heroes he gave them. [...] Capra's social myth, as Robert Sklar has pointed out, required the recognition and participation of the common people to make it come true. It was a myth in which audiences were assured they had a part to play.“ Philip H. Melling: The Mind and the Mob. Hollywood and Popular Culture in the 1930s. In: Philip Davies und Brian Neve (Hg.): Cinema, Politics and Society in America. Manchester 1981, S. 19-40, hier: S. 37.
} 
dem Gemeinwohl unterzuordnen bzw. dies einer Figur zuzuschreiben. ${ }^{90}$ Die Filme stellen mit der Kategorie das soziale Miteinander und die moralischen Gefühle in den Vordergrund. Sie bezeichnet Szenen, in denen eine Figur dazu gebracht wird, etwas zu überdenken oder lernt, sich an Gegebenheiten anzupassen. Die Figuren nehmen dabei die Positionen von Erzieher und Kind ein. ${ }^{91}$

In IT HAPPENED ONE NIGHT wird Ellen (Claudette Colbert) als Person gezeichnet, die für sich Sonderrechte beansprucht. Im Laufe des Films erzieht Peter (Clark Gable) sie, zeigt ihr, mit wenig auszukommen, anderen den Vortritt zu lassen oder zu helfen. So ist es etwa die spezielle Kadrierung, welche das SichNicht-Einfügen anschaulich macht, z.B. als sich Ellen aufgebracht bei einem Busbahnhofs-Angestellten beschwert, dass ihr Bus ohne sie abgefahren sei. In der Einstellung sieht man den Angestellten in der Mitte, mit dem Rücken zur Kamera und Ellen neben ihm, auf der anderen Seite den sitzenden Peter, der die beiden amüsiert beobachtet. Durch die Kadrierung liegt der Fokus weniger auf der aufgebrachten Ellen als auf dem Akt des Beobachtens ihrer Rede. Eine Zwischeneinstellung betont dies noch, darin sieht man Peter näher, wie er das Geschehen verfolgt. So hat man den Eindruck, als ob Ellens Verhalten bereits erwartet wurde. Die Montage agiert hier im Modus des kommunizierenden Gesichts. Man sieht nicht primär das individuelle Verhalten Ellens, sondern die Beobachtung eines Einzelnen in einer sozialen Rahmung. Dieses Registrieren wurde in den Szenen vorher immer wieder vorbereitet. Immer wieder sah man ein Verhalten Ellens, das nicht alleine für sich stand, sondern durch Clark Gables Gesicht kommentiert wurde. Lächerlichkeit, Genugtuung aber auch Schadenfreude kommen durch solche Einstellungswechsel und schauspielerischen Aktionen zustande. Filme wie PLATINUM BLONDE, THE BRIDE CAME C.O.D., MY MAN GODFREY oder THE PHILADELPHIA STORY reflektieren immer wieder eine erzieherische Maßnahme als moralische Genugtuung.

\section{Spiel, Maskerade und Bühne}

Das Thema der sozialen Maske oder gesellschaftlichen Bühne wird von den meisten Screwball Comedies reflektiert. Die Kategorie zielt auf das Gesellige

90 Wie Sennett schreibt: ,[T]he heroine could dream of a large bank account, but she had to learn to live with a small or nonexistent one.“ Sennett 1973, S. 23; vgl. Willet 1996, S. 144.

91 Cavell 1981, S. 84. 
und Gemeinschaftliche in den Komödien ab, auf das Spiel mit Rollen. ${ }^{92}$ Die erste Form, wie die Filme dieses Thema bearbeiten, liegt darin, dass man an den Figuren innerhalb der Diegese eine Verstellung erkennt. Ein Schauspiel zweiter Ordnung wird sichtbar. ${ }^{93}$ Die zweite Form, wie die Filme sich mit dem Thema beschäftigen, ist die Art und Weise, wie der Film selbst - ob im Zusammenhang mit dem Production Code oder aus anderen Gründen - eine Art Maskerade etabliert, nicht das Eigentliche darstellt, sondern ein Thema indirekt anspielt, verschleiert, umschifft und doch erahnbar werden lässt. ${ }^{94}$

Demnach etablieren die entsprechenden Szenen oftmals zwei Szenarien. Historisch leitet sich dieses Prinzip der Bühne, des Spiels und der Maskerade aus dem „sight gag“ ab, wie Noël Caroll ihn für den Slapstick der 1910er und 1920er Jahre beschrieben hat. Er beobachtet die Form des visuellen Humors anhand eines Beispiels aus THE 39 STEPS $^{95}$ : Man sieht das Paar durch Handschellen miteinander verbunden, doch gegenüber der Landlady der Pension gibt es vor ein frisch verliebtes Ehepaar auf Hochzeitsreise zu sein, das die ganze Zeit Händchen hält. Die Konstellation ist so als entspringe sie der Screwball Comedy. Man sieht ein Oszillieren: der Schein des frisch verliebten Paares, doch die Landlady scheint nicht zu hören, wie die beiden Figuren hässliche Worte miteinander austauschen, einander beleidigen und rügen. Carroll schreibt:

Our amusement is generated by the fact that the scene is staged to show not only what is actually going on but what the set of events could also visually support an alternative, and in this case conflicting, interpretation. And it is this play of alternative, often conflicting interpretations, rooted first and foremost in the visual organization of the scene, that primarily causes the amusement that attends sight gags. ${ }^{96}$

In der Kategorie entwerfen die Filme oft zwei Wahrnehmungsräume, auf denen verschiedene Fokalisierungen basieren. Ähnlich wie im Suspense geht es um den Akt, dass man als Zuschauer das Filmbild in der Zeit aufspaltet in zwei Wahrnehmungsweisen, z.B. in sichtbare oder vorgestellte Perspektiven, die erst im letzten Schritt auf die narrative Konstruktion einer Figur bezogen werden bzw. dann als Figurenwahrnehmung oder -wissen verstehbar sind. Man erkennt eine Verstellung als Schauspiel zweiter Ordnung, sieht jedoch, dass die anderen Figuren

92 Siehe auch Glitres Genrekonventionen der Verkleidung, Irreführung, des Betrugs und Rollenspiels mit verschiedenen Identitäten. Glitre 2006, S. 18.

93 Wie in Kapitel 4.2 entwickelt.

94 Seeßlen 1976, S. 28.

95 THE 39 STEPS (Alfred Hitchcock, UK 1935).

96 Carroll 1991, S. 26. 
blind für diese Verstellung $\mathrm{zu}$ sein scheinen. Diese Anordnung ist weniger als abstrakte Informationsvergabe zu verstehen, denn als konkrete Konstruktion von Sichtweisen und Zuschreibungen von Perspektiven, Mitsichten.

Die bühnenhafte Aufführung ist mit einem kindlichen Versteckspiel vergleichbar: In einer Szene aus MR. AND MRS. SMITH ${ }^{97}$ ist David (Robert Montgomery), der sich frisch von seiner Frau Anne (Carole Lombard) getrennt hat, wieder als Junggeselle unterwegs. Er hat ein Blind Date, das sein Freund für ihn arrangiert hat. Nachdem er sich durch die Enge des Clubs geschoben hat, vorbei an tanzenden Leuten, sieht man, wie er etwas (im Off) eingehend betrachtet. Dann sieht man einen point-of-view-Schuss auf eine wunderschöne Frau, die kühl und unnahbar Richtung tanzender Menge schaut. Neben ihr sein Freund, der ihm zuwinkt. Der Kader fährt aus der Zweieransicht an das Gesicht der schönen Frau heran und verweilt dort eine Weile. Aus dem Off hört man die Stimme seines Freundes: „no, not here David“. Man sieht Davids erstauntes Gesicht im Gegenschuss, dann eine Einstellung, die seinen Freund mit zwei etwas älteren Frauen zeigt. Ebenso wie vorher die kühle Schöne werden nun die Frauen zu zweit in einer erneuten point-of-view Einstellung mit leichter Heranfahrt fokussiert. Die Frauen kauen an Selleriestängeln, während sie ihn begrüßen, rauchen und trinken; ihre Kleidungsstücke sind detailreich geschmückt, mit goldenen Verzierungen, Rüschen und Pomp. Durch die enthüllende Montage entpuppt sich die Schöne als Nachbarin am Nebentisch. Allein durch kinematographische Mittel im Zusammenhang mit Sprachäußerungen wird so ein Denkvorgang anschaulich gemacht, der die Annäherung an die Schöne als Illusion und Irrtum ausweist: Der metaphorische Begriff ,Blind Date‘ kommt nur am Anfang der Szene vor. Er wird inszenatorisch ausgearbeitet, wodurch die Metapher auf ihren wörtlichen Ursprung zurückgewiesen wird, was ,blind' in diesem Zusammenhang bedeutet, wird in der Filmszene für Zuschauer expliziert und sinnlich erfahrbargemacht, durch einen Akt des falschen oder nur halben Sehens.

Wenig später sieht man, wie seine Exfrau Ann mit ihrem Begleiter an einem Tisch auf der anderen Seite des Tanzsaals Platz nimmt. Eine Montagesequenz visualisiert, wie beide, David und Ann, einander bemerken, aufeinander achtgeben ohne jedoch einen direkten Blickwechsel auszutauschen oder sich gar zu begrüßen. Ann fragt ihren Begleiter, mit welcher Dame David im Club sei. Wie als würde die Montage direkt auf ihre Frage (,I wonder who he’s with“) antworten, sieht man David, die Kamera fährt dynamisch zurück, zieht auf und so sieht man in einer frontalen Dreiereinstellung David in der Mitte, links von ihm seine an

97 TC: 0:46:29-0:53:18. 
Hühnerbeinen kauende Begleitung und rechts die schöne Unbekannte. Dann folgt ein Montagewechsel, der den Akt des Abwägens symbolisiert, man sieht erst David mit seiner Begleitung in der Zweierkadrierung, dann David mit der Schönen in einer Zweieransicht. Und wie als hätte David die Worte seiner Exfrau gehört, lehnt er sich leicht zurück, hinter die Schöne, beugt sich - ohne dass diese es bemerkt - näher an sie heran. Er beginnt mit theatralischem Gesichtsausdruck zu sprechen, doch stimmlos, so als flüstere er der Schönen etwas zu. Alles an seiner Haltung bedeutet eine Vertraulichkeit, doch stark posierend: seine Lippen formen überdeutlich Worte, die man nicht hört, sein Blick ruht zärtlich auf ihr, doch diese scheint ihm nur zuzuhören. Hinzu kommt, dass die Einstellung auf die Schöne (diesmal mit David zusammen) erneut als ein point-ofview konstruiert wird, diesmal jedoch als Annes Blick. So entsteht das Bild eines gespielten Verhaltens, bei dessen Herstellung man selbst zuschauen konnte, das eigens für den Blick der geliebten Exfrau bestimmt zu sein scheint. Als Zuschauerin versteht man, dass er ein Spiel aufführt, damit Anne denken möge, er sei mit der Schönen zusammen in dem Nachtclub. Doch dieses Denken ist allein in der Montage begründet und zwar in der Art und Weise, wie sich die Worte auf die Kadrierungen und das Schauspiel beziehen. Was sich in der Zuschauerwahrnehmung einstellt ist die Illusion einer Situation, wobei man die Illusionierung in ihrem Entstehen selbst beobachten kann.

Nicht allein das offensichtliche übertriebene Spiel im Schauspiel ist dafür ausschlaggebend, vielmehr sind es vor allem die Kaderbewegung und Montage, welche die Denkvorgänge versinnlichen. Der erste Point-of-view auf die Schöne ist entscheidend, gleich darauf erfährt man die Bezogenheit auf die schöne Frau schon zu Beginn der Szene als verfehlten Blick (,blind'). Im Durchlaufen des ersten Point-of-views und seiner Brechung wenig später in der Montage, etabliert sich so etwas wie ein Muster des illusionären Blickens. ${ }^{98}$ Sobald David sich dann beim auf Annes Sehen bezogenen Point-of-view zur Dame rüberbeugt und eine Vertrautheit spielt, wird dieses Muster gefestigt. Daher ist es weniger ein Wissen darüber, dass die schöne Unbekannte nicht zu der Gruppe gehört als eine kinematographische Wahrnehmung, die unmittelbar ein dynamischer Denkprozess ist und die ein Wissen über die Figuren erst im allerletzten Schritt generiert.

98 Dana Polan erkennt in der Art und Weise, wie in der Szene Blickstruktur aufgebaut ist (,sexuality as battle of gazes“), die enge Einbindung des Films in das Oevre Hitchcocks. Vgl. Polan 1991, S. 134. 
Die etablierte Bühne ist das zentrale Mittel der Komödien, es kann das Spielen einer Rolle, des anderen Geschlechts, einer fiktiven Handlung oder Situation sein (z.B. in PHILADELPHIA STORY, TWENTIETH CENTURY, THE LADY EVE, MIDNIGHT, MR. AND MRS. SMITH, THE TALK OF THE TOWN ${ }^{99}$, I WAS A MALE WAR BRIDE). ${ }^{100}$

In manchen Filmen führt das Inszenierungsprinzip des Verkleidens und des Illusionierens als Bühne soweit, dass sie sich von den Schauspielern löst um ganz filmische Maskerade zu werden. Dann spielt der Film auf etwas an, zeigt es aber nicht, das gilt zum Beispiel für die Darstellung von Sexualität, die aufgrund des Production Codes nicht offensichtlich werden durfte. Eine solche filmische Maskerade wird zum uneigentlichen Sprechen und Zeigen mit allegorischen Zügen. ${ }^{101}$ Sie läuft nur auf der Ebene der Zuschauerwahrnehmung ab und ist kaum mehr an eine nachvollziehbare narrative Handlung gebunden, in der die Verstellung motiviert ist.

So ist die Szene aus MR. AND MRS. SMITH, die vom nächtlichen Champagnertrinken handelt, so aufgebaut, dass sie nur noch in einem übertragenen Sinn funktioniert. Denn das, was auf bildlich-konkreter Ebene erzählt wird, löst sich nicht mehr in einem kongruenten Erzählen auf. Die Szene kreist um den möglichen Sexualakt, der - da Anne und David nicht mehr verheiratet sind für Anne zu einem Skandal wird. Zu Beginn sieht man David, wie er die Champagnerflasche vorbereitet, er dreht sie auf Eis und pfeift fröhlich dazu. Daraufhin sieht man Lombards fragendes und abwartendes Gesicht. Die Dialoge stellen sich ebenfalls in ihrer ,Uneigentlichkeit‘ dar, wenn David sie um die Gläser bittet, sie das wiederholt und dann die Handlung ausführt, doch keineswegs in einer alltäglichen Art und Weise, sondern lauernd-beobachtend und in der Artikulation künstlich-gespielt. Auch als David und Anne mit den Gläsern auf dem Weg zum Schlafzimmer sind, zeigt sich die Atmosphäre (durch die musikalischen Wechsel als auch durch den verstörten Gesichtsausdruck Lombards) als höchst artifiziell. „Keep going, Annie“ raunt David ihr zärtlich zu. Schließlich bittet er Anne die Flasche weiter zu drehen: „Keep it twirling“, was sie tut. Die distanzierte Art und Weise ihres Spiels zu der Flasche führt plötzlich die Anführungszeichen der Inszenierung vor. Schließlich lässt sie vom Drehen der Flasche ab und blickt angewidert auf ihre Hände. Spätestens zu diesem Zeitpunkt tritt deutlich das Allegorische der Handlung hervor: Das nächtliche Vorbereiten des Champagnertrinkens wird als Allegorie auf den sexuellen Akt verstehbar. Als Anne hinter verschlossenen Türen die Flasche

99 THE TALK OF THE TOWN (George Stevens, USA 1942).

100 I WAS A MALE WAR BRIDE (Howard Hawks, USA 1949).

101 Zur Allegorie siehe Plett 1991, S. 90-91. 
zerbricht, fügt sich die Bildbedeutung zur verbalen Bedeutung. Anne schreit: „We're not married! You didn't tell me! - How long could you have waited?!“

Das Allegorische der Szene ist, dass man einen zweifachen, voneinander verschiedenen Sinn konstruiert. Einerseits erlebt man die repräsentierte Handlung selbst als völlig banal: Das Champagnertrinken wird vorbereitet, doch wie aus dem Nichts schreit sich das Paar an. Der Satz Annes ist auf dieser Ebene nicht mehr nachvollziehbar. Andererseits erlebt man, wie die alltäglichen Handlungen Sekunde für Sekunde zu einer allegorischen Handlung ausdeutbar werden. So mag man den Akt des Champagnertrinkens als sexuellen Akt, die Flasche als Phallus und die Interaktion beider als Liebesspiel interpretieren.

Anders als Metaphern in Filmen, welche auf der Basis verschiedener Bildund Erfahrungsbereiche das Verstehen eines Gedankens, einer narrativen Handlung oder einer Figurenkonstruktion in Gang setzen, ist alles am Ablauf der allegorischen Szene uneigentlich. Der Streit löst sich nicht kongruent in ein Erzählmuster auf. Die Allegorie deutet stärker als die Metapher auf eine höhere Ebene, die allein in der Sinnerschließung, im Deutungshorizont selbst hervortritt, während eine erste, einfache Ebene (die in der Sprache der wörtlichen Ebene entspricht) bestehen bleibt.

Die Gefühlsmodi, die mit der Kategorie verbunden sind, bezeichnen sehr häufig, vor allem in ihrer Ausprägung des Schauspiels hoch zwei ein Wahrnehmen von Intimität und Vertrautheit. Dabei ist es meist das Paar, das miteinander ein Spiel aufführt, während andere Figuren außen vor bleiben. Doch nicht die Figuren sind vertraut. Vielmehr eignet sich die Zuschauerin selbst ein Sehen von Vertrautheit als Gefühl im Vorgang der Fiktionalisierung an und überträgt dies wieder auf die Körper im Bild. In der zweiten Form geht es um die komplexe Bedeutungsherstellung, eine Lust am Deuten und Teilhaben an einer Sinnkonstruktion. Dabei ist es weniger das Dargestellte, welches das Vergnügen befördert als die subtile und verschlungene Art und Weise einer ,uneigentlichen` Darstellungsweise, welche amüsiert.

\section{Öffentliche Geste und Entblößung}

Die Kategorie Öffentliche Geste und Entblößung ist komplementär zur Kategorie Bühne, Spiel und Maskerade. Es geht um ein peinliches Verhalten, das Aufsehen erregt, über das sich eine Gruppe von Menschen empört oder belustigt. Soziale Gefühle, die mit gesellschaftlichen Codes, Regeln, Ordnungen und Gesetzen verbunden sind, werden thematisiert. Oder wie James Feibleman sagt: „comedy resides in the contrast between what is and what 
ought to be“. ${ }^{102}$ Das, was in einer bestimmten Situation als angemessenes oder unangemessenes Verhalten angesehen wird, wird von den Filmen immer wieder ausgelotet, gefestigt oder auch kritisch hinterfragt. ${ }^{103}$ Oft sieht man eine Szene, in der zunächst eine gewisse Ordnung oder Ruhe herrscht - dezente Gespräche, zurückhaltende Kommunikationsformen- , man tanzt oder führt Small Talk. Dann kommt es zu einem peinlichen Verhalten. Daraufhin blickt der ganze Saal auf, Passanten auf der Straße laufen zusammen, tuscheln oder rollen mit den Augen.

So gibt es etwa in THE AWFUL TRUTH eine Ballszene. ${ }^{104}$ Darin führt die Frau, die mit Grant ausgegangen ist, eine Bühnenshow auf, in der sexuelle Anspielungen durch ihre Körperbewegungen sehr eindeutig werden: $\mathrm{Zu}$ dem gesungenen Satz „My dreams are gone with the wind“ sieht man, wie eine Windmaschine ihren Rock nach oben wirbelt. Dabei ordnet die Montage zwischen die Bühnen-Performance permanent Einschübe mimischer Reaktionen der zuschauenden Gruppe (Grant, Bellamy und Dunne). Zentral ist hier, dass ihr Tanz nicht auf das einzelne Gesicht eines Zuschauers bezogen wird, sondern auf eine Gruppenansicht, in der verschiedene mimische Reaktionen sich zusammensetzen: Grants Nach-Unten-Blicken und die Handbewegung, welche kurz die Augen verdeckt, Dunnes peinlich berührtes Lächeln (Abb. 44).
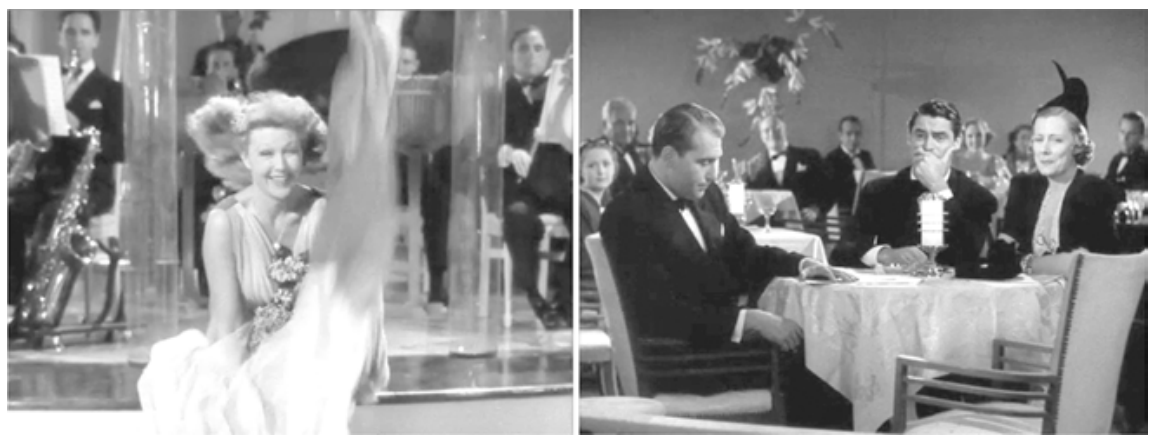

Abbildung 44: Mimische Kommentierung zwischen Peinlichkeit, Schamgefühl und Schadenfreude in THE AWFUL TRUTH.

102 James Feibleman: In Praise of Comedy. A Study in its Theory and Practice (1938). New York 1962, S. 107. Zitiert nach Nowak 1991, S. 16.

103 „[...] die unaufdringliche komische Spannung zwischen förmlichem Benehmen und konventionswidrigen Situationen“, Furler 1990, S. 127-128.

104 TC: 0:25:21-0:35:18. 
Das Komische in der Szene besteht nun nicht in der naiv-freizügigen Tanzweise der Dame, sondern man sieht diese Handlung immer schon bezogen auf die Gruppen-Reaktionen, wodurch die Handlung durch die Mimiken bewertet oder beurteilt erscheint. Das Gefühl der Peinlichkeit kommt nicht durch die Aktion selbst zustande, sondern durch eine reflexive Form in der Montage: Sie ist immer schon mit einer sozialen Rahmung versehen, die sich allein durch die kinematografische Form bestimmt. Montagen sind oftmals so aufgebaut: Man sieht eine Aktion und man sieht Gesichtsbilder oder eine Menge oder Gruppeanansicht, welche dies missbilligend, amüsiert oder empört betrachtet.

In der Gruppe der Öffentlichkeit sind zudem manchmal Gesetzesvertreter, Ordnungshüter und offizielle Personen vertreten, die eine Institution verkörpern. Diese Anwesenheit des Gesetzes oder des Offiziellen stellt immer wieder die soziale Rahmung des Paares her, das Überwachende in den Komödien man könnte auch sagen, der Production Code - schreibt sich in die Komödien als narratives Element ein. Stets wird in einer Szene die Anwesenheit des Gesetzes mit dem Paarsein und den ehelichen Handlungen - Übertritte und Fehlverhalten - verbunden (Abb. 45).
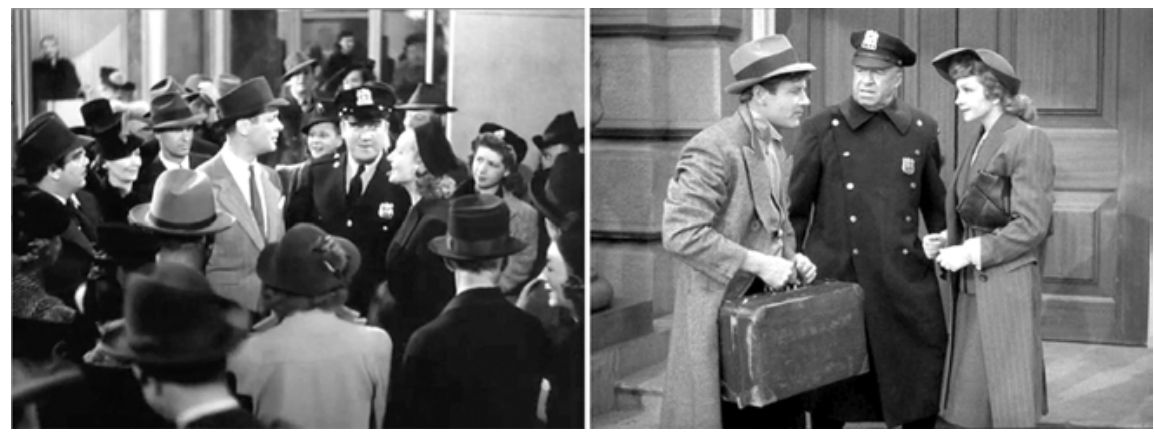

Abbildung 45: Der Gesetzeshüter und das Paar in MR. AND MRS. SMITH (links) und THE PALM BEACH STORY (rechts).

Die Anwesenheit einer Öffentlichkeit, in der Privates diskutiert wird, kann jedoch auch als Einbettung von Darstellungen von Medien vorkommen, z.B. sieht man das Insert eines Zeitungsartikels. ${ }^{105}$ Diese Dimension hat sich vor allem in

105 Oder wie Cavell sagt: „Der schlichten Einschätzung, dass die Thematik der Filme privater Natur ist, steht die Tatsache entgegen, dass es offenkundig typisch für die Eskapaden des Paares ist, dass sie in die Zeitung kommen." Cavell 2010, S. 69. 
den Filmen ausgeprägt, welche von Journalisten oder öffentlichen Fällen handeln (NOTHING SACRED, IT HAPPENED ONE NIGHT, LOVE ON THE RUN, ONCE UPON A HONEYMOON, LIBELED LADY, THE FRONT PAGE, THE PHILADELPHIA STORY, ADAM'S RIB, HIS GIRL FRIDAY) ${ }^{106}$. Die Filme stellen selbst ein Reflektieren über den Status der Anwesenheit einer bestimmten Öffentlichkeit dar. Oder wie Cavell am Beispiel von THE PHILADELPHIA STORY sagt: „Die Frage, was im Wesentlichen öffentlich und was im Wesentlichen privat ist, wird zu Beginn als ein Problem innerhalb des Films selbst eingeführt. “107 Die Instanz einer bewertenden, ordnungsstiftenden aber auch einengenden Öffentlichkeit wird $\mathrm{zu}$ einem Teil des $\mathrm{Zu}$ schauerblicks. ${ }^{108}$ Die Gefühlsmodi, die sich mit dieser Kategorie verbinden können, weisen das ganze Spektrum negativer, sozialer Gefühle und deren Komponenten auf: Schadenfreude, Scham, Peinlichkeit, das Spüren einer öffentlichen Kontrolle, Mahnung oder Bestrafung. Dabei ist das Wesen der Komödie jedoch so angelegt, dass eine Kategorie meist nicht allein in einer Szene vorherrscht, sondern mit anderen Kategorien zusammenkommt und sich über die Montage rasch abzuwandeln beginnt. Daher werden die negativen Gefühle -Scham, Peinlichkeit - zwar erfahrbar, aber sie werden in der jeweiligen, spezifischen Inszenierung kaum je vertieft, stets haftet ihnen Leichtigkeit und Wandlungsfähigkeit an.

\section{Die Einheit des Paares}

Innerhalb dieser Kategorie inszenieren die Filme Wir-Gefühle, die jedoch höchst unterschiedlich gestaltet sein können. Die Kategorie Einheit des Paares teilt sich auf in drei Formen. Die erste Ausprägung dient der Inszenierung von Paarbildern, während die zweite Ausprägung eine Variation dessen ist, aber unter den Vorzeichen des Production Codes. Man könnte sagen, in der zweiten Kategorie geht es stärker als in der ersten um Sexualität, die allerdings rein indirekt oder nur angespielt dargestellt wird. Die dritte Ausprägung der Kategorie bringt eine Gruppeneinheit zur Darstellung. Das zentrale poetologische Prinzip dieser drei Ausprägungen als Familienähnlichkeit lässt sich wie folgt kurzfassen: Die Komödien präsentieren oftmals eine höchst widersprüchliche Einheit

106 NOTHING SACRED (William A. Wellman, USA 1937). LOVE ON THE RUN (Woodbridge S. Van Dyke USA, 1936). ONCE UPON A HONEYMOON (Leo McCarey, USA 1942). LIBELED LADY (Jack Conway, USA 1936). THE FRONT PAGE (Lewis Milestone, USA 1931).

107 Cavell 2010, S. 70.

108 So auch die Anwesenheit der Kamera und die Standfotografie am Ende von THE PHILADELPHIA STORY, vgl. Cavell 2010, S. 71. 
(eine ,Gegensinnigkeit als Einheit ${ }^{609}$ ), wobei die kinematographische Inszenierung die Worte, Stimmbilder, Gesten und Gesichtsausdrücke der Schauspieler so aufeinander bezieht, dass eine Einheit bzw. Gruppen- oder Paarwerdung in der filmischen Wahrnehmung stattfindet. Dabei können übereinstimmende und kontrastive Bewegungsqualitäten zwischen den Schauspielern oder aber zwischen Kamera/Kader/Montage und Schauspielkörper so orchestriert werden, dass sie tendenziell Leichtigkeit, Raschheit, Eleganz, Symmetrie, Synchronie und rhythmische Entsprechungen aufweisen. Die Kategorie ist die zentralste von allen und daher reflektieren alle Filme des Screwball Comedy Genres das Thema der Einheit des Paares in der ein oder anderen Weise.

1) Die erste Form bezieht sich auf eine bildlich-audiovisuelle Vereinigung oder die Anziehung des Paares. ${ }^{110}$ Diese Form meint die kinematografische Gestaltung der Wahrnehmung von Zuschauerinnen, so dass überhaupt die Idee von einem Paar zustandekommt. Meist ist das Paar auf narrativer Ebene getrennt, doch in der Art und Weise, wie die Mise-en-scène, das Schauspiel und die Montage das Paar zueinanderstellt - meist in einem bestimmten Sprech- und Interaktionsrhythmus, der jedoch nicht mehr vom Filmbild und seiner Inszenierung abzulösen ist -, erscheint eine Formation oder Figuration des Paares als körperliche oder kommunikative Einheit. Oder wie Georg Seeßlen sagt:

Jede erotische Komödie beschreibt, wie Mann und Frau ihr emotionales Timing aufeinander einstellen. Die Screwball-Comedies reflektieren dabei die Bedingungen des Eros in der spätbürgerlichen Industriegesellschaft (...). In den Vorgängen, die die Screwball Comedy beschreibt, fällt Komik ab als Derivat erotischer Spannungen. Ihre Protagonisten bedienen sich der Methode des schnellen und anhaltenden Sprechens, welches ein Symbol erotischer Offensive ist (man denke nur an Groucho Marx). Die verwandelnde Erotik des Films offenbart sich in Montage und Tempo; die Bildersprache der Screwball Comedies ist so kompliziert, wie es auch die erotischen Beziehungen sind, die sie darstellen - und nicht nur aus Zensurgründen. ${ }^{111}$

Die Bildersprache der Komödien, die der Kompliziertheit erotischer Beziehungen entspricht, wie Seeßlen sagt, ist entscheidend. Montage und Tempo können die Spannungen des Paares für uns Zuschauer versinnlichen. Dabei werden die Figuren durch ganz unterschiedliche Mittel aufeinander bezogen, sie werden auf der Ebene der Zuschauerwahrnehmung vereint: Eine Figur führt eine Handlung aus, welche durch eine zweite Figur rhythmisch fortgesetzt wird.

109 Plessner 2003 (1945), S. 294.

110 Vgl. Leach 1977, S. 77; Babington und Evans 1989, S. 13.

111 Seeßlen 1976, S. 82-83. 
Oder die Einstellungsgrößen entsprechen einander. Oder es gibt symmetrischgrafische Muster einer Paarwerdung, die Synchronie im Sprechen und im Gestikulieren. Diese sind allesamt Inszenierungen, die das Wahrnehmen von Ebenbürtigkeit und Gleichberechtigung auf rein sinnlicher Ebene erzeugen. ${ }^{112}$ Auch wenn wir Zuschauerinnen ein streitendes Paar erleben, dann erfahren wir zugleich etwas völlig anderes, eine Paargeste, die eine intime Verbindung aufzeigt. Die Kategorie zielt auf das Paarwerden durch kinematografische Mittel, das heißt Szenen werden so montiert, dass sie über die Zeit hinweg auf der Ebene der Zuschauererfahrung die Bilder so arrangieren, dass man die Aufeinanderbezogenheit von verschiedenen Schauspielerkörpern, ein ,Paarsein` überhaupt erst wahrnimmt.

2) In den pre-code Filmen vor 1934 werden Sexualität und außereheliche Partnerschaften offen thematisiert, z.B. im Film DESIGN FOR LIVING von Ernst Lubitsch, in dem zwei Männer und eine Frau abwechselnd miteinander leben, sich lieben, verlassen und wieder zusammenkommen. Mit Beginn des Production Codes verengt sich der Spielraum. Sexualität kann anders noch als wenige Jahre vorher nur noch über indirekte Formen ${ }^{113}$ angespielt bzw. versinnlicht werden. Die Komödien verwenden daher Formen der Indirektheit (z.B. mit metaphorischen Konstruktionen), sie spielen damit, sich an den Regelcode der Filmindustrie zu halten, aber so, dass die Regel eingehalten und konterkariert wird. ${ }^{114}$ So reflektiert etwa der Film IT HAPPEND ONE NIGHT die Verschärfung der Zensur ab 1934, indem er die Anweisung ein Liebespaar nicht in einem gemeinsamen Bett liegend zu zeigen, wörtlich umsetzt. Das unverheiratete, getrennt schlafende Paar hängt zwischen die Betten eine Decke. Eine ganze Weile thematisiert der Film die Art und Weise, wie das Paar sich näherkommt, aber gleichzeitig diese Barriere zwischen ihnen aufrechterhalten bleibt. Dabei wird die Wand bzw. Decke über eine gewisse Zeit filmisch umgedeutet. Stellt sie anfangs die notgedrungene Schicksalsgemeinschaft der beiden dar, in der

112 Die Idee einer ,Ebenbürtigkeit‘ der Geschlechter wird in der Forschungsliteratur immer wieder behauptet, ohne dies an die konkreten Inszenierungsformen rückzubinden. Z.B. Babington und Evans 1989, S. 13.

113 Jane M. Greene beschreibt, wie der Prozess zwischen Zensurbehörde und Filmindustrie sich historisch-dynamisch änderte und stellt fest, dass der Production Code vor allem insofern auf die Drehbuchautoren und Produzenten der Komödien Einfluss nahm, als er bei Anspielungen von sexuellen Themen eine maximale Subtilität forderte. Jane M. Greene 2011.

114 Zum Verhältnis von Production Code und den Screwball Comedies siehe Jane M. Greene 2011. 
das Trennende und die Distanz als Schutz der Frau verstehbar ist, wandelt sie sich in ihrem symbolischen Gehalt zu einem das Paar verbindenden Gegenstand, wobei das Aufhängen der Decke bald zu einem Ritual des Gemeinsamen wird, an dem beide festhalten. Die Art der filmischen Reflexion ist an die Dramaturgie und Expressivität des Films gebunden, an die Art, wie sich eine Metapher in der Zeit des Films ausbildet, die jedoch nicht eine fixe Bedeutung hat, sondern einen Wandel von Bezügen als Denkbewegung anordnet. Diese kann nun allein im Rahmen der einzelnen Filmerzählung situiert werden oder an die in der Filmindustrie auftauchende Verschärfung der Zensur rückgebunden werden. Das Beispiel der fluide sich wandelnden Bedeutung des getrennten Schlafens bildet eine Familienähnlichkeit im Genre aus; spätere Filme bedienen sich einer ähnlichen Art, wie der Deutungshorizont in Bezug auf den Production Code ausgespielt wird. So führt die Szene der getrennten Zimmer (und der hellhörigen Wand) aus MR. AND MRS. SMITH diesen genrespezifischen Strang bildlicher Reflexion fort. Auch das Beispiel aus THE AWFUL TRUTH, in dem die Tür zur Metapher der Getrenntheit wird, die es zu überwinden gilt, ist Teil der gemeinschaftlichen Reflexion der Filme, eine Familienähnlichkeit. Dabei sind nicht Ausstattung oder Dekor entscheidend, sondern die Art und Weise, wie ein Gegenstand durch die Inszenierung zum Sinnbild für die Trennung bzw. Verbundenheit des Paares wird. Das Nachdenken über den Production Code und seine strengen Sittenregeln kann in diesen drei Beispielen als Umwertung verstanden werden. Das Zeigen eines Paares in getrennten Betten oder Räumen wird zwar auf repräsentierter Ebene eingehalten, doch der jeweilige Montagekontext rückt das Getrenntschlafen so in den Vordergrund, dass es sich in seiner Bedeutung zu wandeln beginnt: Es wird zum Sinnbild für das Paar selbst, das freilich am Ende glücklich vereint ist.

3) Die dritte Ausprägung der Kategorie inszeniert die Vereinheitlichung von Gruppenformationen, z.B. über Gesicht- oder Stimmbilder, oft geht es um eine widersprüchliche Gemeinschaftlichkeit. Dabei spielen vor allem die bereits erörterten kontrastiven Emotionsdarstellungen eine Rolle. Eine Montage vereint verschiedene Gesichter in einer Reihe, doch diese streben auseinander und werden doch immer wieder durch die stets gleiche Form der Kadrierung und Montagebewegung in eins gesetzt. ${ }^{115}$

115 Siehe das Bsp. aus BLUEBEARD’s EIGHTH WIFE in Kap. 3.4. 


\section{Alltäglichkeit}

Mit den Screwball Comedies erhält auch ein bestimmter Bereich menschlichen Lebens Einzug in die Filmwelt: Das Gewöhnliche, Alltägliche, das Tag-ein-Tagaus des Ehelebens. Man sieht die Protagonisten alltägliche Handlungen der Routine ausführen, Geschirrspülen (MY MAN GODFREY), eine Mahlzeit zubereiten (IF YOU COULD ONLY COOK ${ }^{116}$, ADAM'S RIB), sich rasieren lassen (MR. AND MRS. SMITH) oder sich langweilen (THAT UNCERTAIN FEELING). Zwar sind auch viele Komödien von der Inszenierung des Spektakulären, eines Freizeitverhaltens der Reichen geprägt - man fliegt mit dem Privatjet (THE BRIDE CAME C.O.D. ${ }^{117}$ ), geht zum Pferderennen (THE AWFUL TRUTH), kauft haufenweise edle Kleidungsstücke (THE PALM BEACH STORY), verbringt Zeit in teuren Hotels (EASY LIVING) oder amüsiert sich auf einem Kreuzfahrtschiff (THE LADY EVE, HOLIDAY) -, doch neben diesen Ausstellungen des spektakulären Freizeitvergnügens, die oftmals mehr an die Kategorie der Vision eines reichen Lebens gebunden sind, ist im Kontrast dazu das Gewöhnliche vor allem durch den Einzug des Alltagsgesprächs zu verzeichnen. ${ }^{118}$ Menschliche Sozialität wird durch die Inszenierung vielfältiger Face-toFace-Kommunikationen dargestellt: Wie gesprochen wird, wie man Blicke austauscht, wie man auf ein unangemessenes Verhalten reagiert. Die Kategorie stellt die Alltagshandlungen und Gewohnheiten dar. Alltagshandlungen laufen meist parallel zu den Unterhaltungen ab. So wie etwa in MY MAN GODFREY, als Irene (Carole Lombard) ihrem Butler Godfrey (William Powell) eine Liebeserklärung macht, während sie zusammen die Küche aufräumen. Das Gespräch ordnet sich um ihre Verliebtheit und um seine höfliche Zurückhaltung. Dabei sieht man die Handlung des Spülens ziemlich ausgedehnt. Während sie spricht, sieht man, wie die Millionärstochter Irene (Carole Lombard) ungeschickt im Abtrocknen ist, sie fasst die Teller so an, als verrichte sie das erste Mal in ihrem Leben Hausarbeiten. Hinzu kommt, dass sie die Zärtlichkeit ihrer schmachtenden Worte stets ein bisschen in ihre Handbewegungen legt und so auch an Teller und Spültuch weiterzugeben scheint. Ganz im Gegenteil dazu sieht man die Bewegungen des Schauspielers Powell stets als souverän, routiniert, ruhig und sicher. Die lange Ausstellung des Tellerwaschens dient jedoch nicht nur der Betonung der Alltäglichkeit, sondern lässt sich wiederum auf den Geschlechterdiskurs der Komödien beziehen, der oftmals Klischees unterläuft bzw. karikiert. $\mathrm{Zu}$ den Ritualen des Alltags gehört auch das gemeinsame Arbeiten in einem

116 IF YOU COULD ONLY COOK (William A. Seiter, USA 1935).

117 THE BRIDE CAME C.O.D. (William Keighley, USA 1941).

118 Siehe Kapitel 3.2. 
Büro, z.B. in den Komödien, welche das Paar als Kollegen entwirft (HIS GIRL FRIDAY, PLATINUM BLONDE, TROUBLE IN PARADISE ${ }^{119}$ ) oder in einem Angestelltenverhältnis zeigt (DANGER - LOVE AT WORK, HANDS ACROSS THE TABLE, SHE MARRIED HER BOSS) ${ }^{120}$. Die langsamen Gespräche sind gewissermaßen komplementär zu den rasanten Konversationen im Modus des Schlagabtauschs. Anders als bei diesen, deren Fokus auf der Einheit des Paares liegt, schaffen jene durch ihr Andauern oder die Wiederholung einen affektiven Modus der Kontemplation und Vertrautheit.

\section{Das Dreieck}

Mit der Kategorie Das Dreieck werden Themen wie Treue, Eifersucht und Ehebruch reflektiv bearbeitet. Sie bildet eine Gegenfigur zur Kategorie der Einheit des Paares, können aber auch mit ihr zusammen auftreten, wenn es innerhalb einer Dreierkonstellation ganz offensichtlich eine Paarinszenierung gibt. Außereheliche Beziehungen waren bereits in den Scheidungs- und Gesellschaftkomödien der 1920er Jahre ein Topos, bei DeMille und Lubitsch. ${ }^{121}$ Für das Screwball Genre manifestiert sich die Kategorie als erstes bei Lubitsch, in den Pre-Code-Filmen TROUBLE IN PARADISE und DESIGN FOR LIVING, in denen eine ménage à trois zum Beziehungsmodell wird. ${ }^{122}$ Das Thema wird in den meisten Filmen aufgegriffen und bearbeitet, wenn auch nicht in der Offenheit wie bei Lubitsch. Meist ist eine Trennung oder Scheidung vollzogen worden, weshalb es eher um den neuen (und falschen) Partner geht. Es gibt Filme, welche um die Legitimität von Beziehung ringen, ob die Ehe nun aus Versehen annulliert wurde (MR. AND MRS. SMITH), oder ob der Partner nach Jahren, in denen er oder sie verschollen war, zurückkehrt (TOO MANY HUSBANDS, ${ }^{123}$ MY FAVORITE WIFE $\left.^{124}\right)$. Die Eindeutigkeit, die DESIGN FOR LIVING an den Tag legt, wird allein durch TOO MANY HUSBANDS erneut aufgegriffen. Der Film bearbeitet zudem die Frage, wie man Bi- und Homosexualität in den Zeiten des Production Codes

119 TROUBLE IN PARADISE (Ernst Lubitsch, USA 1932).

120 DANGER - LOVE AT WORK (Otto Preminger, USA 1937). SHE MARRIED HER BOSS (Gregory La Cava, USA 1935).

121 Siehe Musser 1995.

$122 \mathrm{Zu}$ letzterem schrieb die Filmkritik 1933: „,Design for Living“ sets a new code for women as Gilda finds her heart large enough to give employment to two boyfriends.“ Motion Picture Herald, 2. Dezember 1933, S. 45, zitiert nach Andrew Bergman: We're in the Money. Depression America and its Films. New York 1971, S. 59.

123 TOO MANY HUSBANDS (Wesley Ruggles, USA 1940).

124 MY FAVORITE WIFE (Garson Kanin, USA 1940). 
thematisieren kann (der Film ist gleichzeitig der Kategorie Maskerade, Spiel, Bühne zugeordnet). Immer wieder bildet sich in dem Film das Männer-Paar zu einer Einheit aus, während die Frau in den Hintergrund tritt. Das Männerpaar schläft in einem Zimmer, trägt ähnlich plüschige Pyjamas und ist den ganzen Film über damit beschäftigt, miteinander zu ringen, sich $\mathrm{zu}$ messen und $\mathrm{zu}$ vergleichen. Zudem werden die drei polizeilich verfolgt; die Freizügigkeit, mit der sie zu dritt in einem Haus leben, wird akribisch von einem Ordnungsamt überprüft. Am Ende des Films in einem Tanzsalon zeigt die Kamera überall Paare, wohin man blickt, doch auf der Tanzfläche taucht das Dreiergespann auf, ein ungewöhnliches Happy End. Die Komödien bearbeiten die Fragen nach außerehelichen oder polygamen Beziehungen, die Darstellung von Eifersucht in einem Erfahrungsmodus, der von Zuschauern als Lust an der Koketterie oder Sensation, als Schadenfreude angesichts des Verbotenen, moralisch Verwerflichen oder Grenzüberschreitenden nachvollzogen wird. Freilich lässt sich hier wie in anderen Kategorien auch eine historische Zuschauerschaft anhand des Aufrufens moralischer Zusammenhänge nachzeichnen, die mit den affektiven Adressierungen, die rein bewegungsmäßiger Natur sind, zwar zum Teil identisch zu sein scheinen, wodurch ein historisch gewordenes Gefühl für uns heute nachvollziehbar wird. Andererseits gibt es Darstellungen, deren Gefühlsschattierungen nicht mehr in allen Dimensionen transparent werden. ${ }^{125}$

\section{Geschlechterrollen und Gleichberechtigung}

Mit der Kategorie Geschlechterrollen und Gleichberechtigung reflektieren die Filme, wie die sozialen Beziehungen sich mit der zunehmenden Erwerbstätigkeit der Frauen verändern. Geschlechterrollen und Berufstätigkeit stehen im Zentrum, es wird immer wieder das Thema der Gleichberechtigung aufgeworfen, indem inszenierte Ansichten der Hierarchie oder solche der Ebenbürtigkeit bildkompositorisch eingebunden werden. Die Thematik verweist auch auf das Stichwort des „woman's film“. ${ }^{126}$ Dabei kreisen die Erzählungen darum, dass Konflikte auftauchen, die stets aufgrund der Geschlechterproblematik existieren. Anfang der 1930er Jahre tauchen hauptsächlich die working girls in den Filmen auf, die von den in den 1940er Jahren dargestellten Karrierefrauen abgelöst

125 Zum Verhältnis von Moral und Gefühl im Film siehe Grotkopp 2017.

126 Jeanine Basinger: A Woman's View. How Hollywood Spoke to Women, 1930-1960. London 1993, S. 20. 
werden. ${ }^{127}$ Auch weibliche Intellektuelle sind vertreten, die Bestseller-Autorin in THEODORA GOES WILD ${ }^{128}$, die feministische Schriftstellerin in THE DOCTOR TAKES A WIFE $^{129}$, die Psychoanalytikerin in SHE WOULDN'T SAY YES ${ }^{130}$, die Richterinnen in THE BACHELOR AND THE BOBBY-SOXER und TELL IT TO THE JUDGE ${ }^{131}$ oder die feministische Rechtsanwältin in ADAM'S RIB.

Der Zusammenhang von Geschlechterrollen und Berufstätigkeit erscheint auch über verbale Zuschreibungen, z.B. wenn in HIS GIRL FRIDAY Walter Hildy mehrfach anerkennend als „newspaper man“ bezeichnet. Oder aber das Thema wird über eine Verkehrung der männlichen und weiblichen Mehr- und Minderheitsverhältnisse angespielt, so etwa wenn man Sam (Spencer Tracy) in wOMAN OF THE YEAR ${ }^{132}$ bei einer feministischen Tagung sieht, bei der er als einziger Mann auf dem Podium zwischen einer Reihe von Frauen sitzt. Aus Versehen passieren ihm eine Reihe von Missgeschicken, die das Unbehagen aufgrund der ungewohnten Position verdeutlichen. Damit wird eine höchst erfolgreiche, berufliche Frauenwelt gezeichnet, in der die herrschende Normativität von Geschlechterrollen ausgehebelt zu sein scheint. Manche Filme bringen dies auch mit dem Geschlechtertausch als cross-dressing zusammen (I WAS A MALE WAR BRIDE, BRINGING UP BABY), wobei die Filme dann eine Verbindung mit der Kategorie Spiel, Maskerade und Bühne eingehen.

Cavell hat das Sub-Genre der Wiederverheiratungskomödie auch „comedy of equality“ genannt und erörtert, dass die Frauen in den Filmen als Töchter der Sufragetten erscheinen, dass die Filme eine Phase des Feminismus bezeichneten. ${ }^{133}$ Die Gleichstellung von Mann und Frau entwerfen die Filme auf der Ebene konkreter Bildformationen als Erleben zweier ringender, miteinander

127 So bildete sich in den 1940er Jahren der Filmzyklus des Career Woman's Film heraus, darunter Screwballs, Melodramen und Romantic Comedies. Der Zyklus reflektiert die Veränderung des Frauenbildes mit Eintritt der USA in den Zweiten Weltkrieg, vgl. Ralph Willet: The Nation in Crisis. Hollywood's Response to the 1940s. In: Philip Davies und Brian Neve (Hg.): Cinema, Politics and Society in America. Manchester 1981, S. 59-75, hier: S. 64.

128 THEODORA GOES WILD (Richard Boleslawski, USA 1936).

129 THE DOCTOR TAKES A WIFE (Alexander Hall, USA 1940).

130 SHE WOULDN'T SAY YES (Alexander Hall, USA 1945).

131 THE BACHELOR AND THE BOBBY-SOXER (Irving Reis, USA 1947); TELL IT TO THE JUDGE (Norman Foster, USA 1949).

132 WOMAN OF THE YEAR (George Stevens, USA 1942).

133 Cavell 1981, S. 18-19. Oder Babington und Evans dazu: „Concentration on the heroines of Screwball allows one to see that these films embrace a belief in a fundamental though contradictory tenet of Western bourgeois liberalism, that belief in the equality of men and women formally enshrined in the 1919 Suffrage Amendment [. . .].“ Babington und Evans 1989, S. 13. 
schwingender oder ausgewogener Kräfte, z.B. durch synchrone oder symmetrische Formationen der Bildgestaltung. ${ }^{134}$

\section{Emotionskarikatur}

Das Schauspiel ist in vielen Filmen des Genres als Emotionskarikatur angelegt. Dies liegt immer dann vor, wenn die einzelnen Figuren ein posierendes Mienenspiel, eine exaltierte Geste oder anderweitige Übertreibungen zur Darstellung bringen. Ist dieses Gebaren in ein narratives Spiel eingebunden, paart sich die Kategorie Emotionskarikatur häufig mit der Kategorie Maskerade, Spiel und Bühne. In vielen Fällen tritt die Überzeichnung jedoch auch ohne erzähltechnischen Zweck auf. Auch übertriebene Ganzkörperperformances, die aus den Slapstick-Filmen der 1920er Jahre herrühren, sind Teil dieser Kategorie. Der Zusammenhang von mimischer Emotionskarikatur und ganzkörperlichem Slapstick liegt in einer gewissen Objektgebundenheit. Während der Slapstick meist ein leichtes und heiteres Spiel gegen widerständige und sich verselbständigende Objekte darstellt, wird in der Emotionskarikatur der Gesichtsausdruck selbst objekthaft. Nicht der Affekt in seiner zeitlichen Entfaltung wird sichtbar, sondern das zum Schema vergröberte Antlitz reflektiert sich selbst als ein wiederzuerkennendes Ding. Das zwischenmenschliche Beziehungsgeflecht wird z.B. als maschineller Rhythmus erfahrbar, der weniger an Individuen mit Gefühlen als an ein Reiz-Reaktions-Muster erinnert. ${ }^{135}$ Die Kategorie lässt sich vor allem über den herausgearbeiteten Bildtypus des posierenden Gesichts fassen, beschränkt sich aber nicht darauf. Das Vergnügen liegt in der Attraktion einer Vereindeutigung, Plastizität und Erstarrung des Emotionsausdrucks.

\section{Romantik und Sentimentalität}

Sentimentalität, Sehnsucht und Rührung tauchen immer wieder auf in den Filmen der Screwball Comedy, wenn auch meist nur für kurze Augenblicke und daher mehr als Andeutung denn als affektpoetisch wirksame Kategorie. In den Filmen von Frank Capra (insbesondere IT HAPPEND ONE NIGHT) ist die Kategorie Romantik und Sentimentalität prominenter als in den anderen Screwball Komödien. Während die Kategorie Einheit des Paares mit dem Gemeinschaftlichen beschäftigt ist, rückt

134 Vgl. Kapitel 4.4.

135 Seeßlen 1976, S. 49-50. 
mit der melodramatischen Inszenierung das individuelle Empfinden, die Sehnsucht oder das Verlustgefühl in den Vordergrund. Der melodramatische Erfahrungsmodus visiert das überhöhte Idealbild, das ersehnte Abwesende, Beweinte, Traurige und Sehnsuchtsvolle an und lässt sich als Zeitstruktur im Zuschauerempfinden beschreiben. Dies ist im Melodrama an die „Zeit des Sichtbarwerdens der Gefühle“136 gebunden, die Dauer gestaltet für den Zuschauer einen Akt der Verinnerlichung. Die sich entfaltende Geste der Heroine und das langsame sich Entwickeln des Filmbilds konstruieren eine expressive ästhetische Form: ein Empfindungsbild. ${ }^{137}$ Im Rahmen der Screwball Comedy ist die Kategorie jedoch nur eine Andeutung des melodramatischen Modus. Die Familienähnlichkeit ist immer nur für kurze Momente anwesend und anders als in anderen Genres bzw. im Melodrama nicht in einer Dauer ausgestellt. ${ }^{138}$ Wie am Beispiel von HIS GIRL FRIDAY gezeigt, stellen die im Modus des Melodramatischen orchestrierten Ausdrucksbewegungseinheiten der Eingangsszene des Films die ernsten Augenblicke dar, die eine wichtige Funktion einnehmen für die heiter-beschwingten Modulationen. Sie zentrieren und gewichten das Ansichtigwerden der Paarbeziehung. Für einen Moment tritt das individuelle Empfinden hervor, wird in den audiovisuellen Kompositionen sichtbar und verdrängt die vorherrschende Pluralität. In der Zuschauerwahrnehmung wird länger eine Figur ins Zentrum gerückt, deren Gesichtsbewegung oder Stimmklang dauert länger an. Der Wechsel des melodramatischen und komödischen Modus $^{139}$ bewirkt eine Verbundenheit des Zuschauers mit den Figuren, ein Changieren zwischen dem Selbstempfinden und der dyadischen Spannung.

\section{Die Idee des Genres}

Grundsätzlich stellen alle Szenen aller Screwball Comedies in ihrer Gesamtheit die Idee des Genres dar. Die Kategorien dienen weniger als prototypische Szenenanordnungen, denn als affektpoetische und narrative Elemente. Nicht ein Muster wird wiederholt durchgeführt oder variiert, sondern eine bestimmte Idee oder ein Thema, wie Cavell sagt, wird von den Filmen gemeinsam reflektiert.

136 Kappelhoff 2004a, S. 157.

137 Zum Begriff siehe Kappelhoff 1998.

138 Zur Gegenüberstellung von melodramatischem und komödischem Erfahrungsmodus siehe Kappelhoff und Greifenstein 2014, 2017.

139 Für das sentimentale Genießen ist ein Prozess der Verinnerlichung ausschlaggebend. Kappelhoff 2004a, S. 21-22. 
Dieses Thema oder die Idee ist ein vielfältiges Mosaik, das verschiedene Aspekte der Paarbeziehung der bzw. Ehe beleuchtet: Die Kategorie Einheit des Paares steht im Mittelpunkt. Ihre Gegenkategorie ist Das Dreieck, welche die Einheit gefährdet. Sie erfährt eine soziale Rahmung durch die Kategorie Spiel, Maskerade und Bühne. Die Kategorie Öffentliche Geste und Entblößung bezieht sich auf Verstöße gegen normative Setzungen und Maßregelungen des ehelichen Verhaltens durch das soziale Umfeld oder Ordnungshüter bzw. staatliche Institutionen. Ehe-interne Spannungen und Hierarchien werden durch die Kategorien Erzieherische Maßnahme und Geschlechterrollen und Gleichberechtigung in den Blick genommen. Alle aufgeführten Kategorien haben narrative und affektpoetische Funktionen, außerdem dienen sie dazu, ein bestimmtes Nachdenken zu den Bedingungen, Gegebenheiten und Rahmungen von Paarbeziehung im kulturell-historischen Setting der USA der 1930er und 1940er Jahre offenzulegen, hier ist besonders die Kategorie Vision eines reichen Lebens angesprochen. Die Kategorien Alltäglichkeit, Emotionskarikatur und Romantik und Sentimentalität stellen hingegen hauptsächlich stimmungsbezogene Kategorien dar.

Alle Kategorien sind jeweils als affektive Dimension beschreibbar. Sie reichen von sozialen Gefühlen der Scham, Schadenfreude und Peinlichkeit (z.B. in Öffentliche Geste und Entblößung) über Freude und Lust (in Das Dreieck, Die Einheit des Paares), bis hin zu kurzen Momenten der Sehnsucht (Romantik und Sentimentalität).

Man kann dies an einem Beispiel gut veranschaulichen: So ähneln THE AWFUL TRUTH und HIS GIRL FRIDAY einander dadurch, dass sie fast dasselbe SchauspielEnsemble vereinen, was Cavell „the recurrency of faces“ genannt hat. Man sieht in beiden Filmen Cary Grant und seinen Gegenspieler Ralph Bellamy, nur die Frauenfigur ändert sich jeweils (Irene Dunne bzw. Rosalind Russell). Doch darüber hinaus werden mit den Schauspielern ähnliche Charaktere gezeichnet. Außerdem reflektieren sie gemeinsam die Einheit des Paares als subtile Verbindung, sowie das Dreieck. In HIS GIRL FRIDAY gibt es eine Szene, in der Walter, Hildy und Bruce miteinander essen gehen. In der Szene, in der sie in einem Restaurant sitzen, greift die Montage stets die Gemeinsamkeit von Walter und Hildy hervor; die Kadrierung fokussiert stets eine Inszenierung von synchronen Gesten und symmetrischen Bildanordnungen, die an die Schauspielerkörper Grants und Russels gebunden sind. Durch die Tempowechsel und Rhythmik von Montage, Dialog und Schauspiel erfährt man eine Paareinheit (Walter und Hildy) sowie eine bildlich randständige Position (Bruce). Eine Spiegelszene dazu stellt in THE AWFUL TRUTH die Szene an der Tür dar (wie in Kapitel 5 beschrieben). Hier ist es ebenso, dass der Montagerhythmus eine Paareinheit generiert - durch match cuts und symmetrische sowie synchrone Kompositionselemente - und eine randständige 
Figur, die man oft aus dem Off sprechen hört, die aber niemals zum Zentrum der Montageeinheit wird. Beide Szenen bilden insofern Familienähnlichkeiten aus, als dass sie nicht nur dieselben Schauspieler ins Bild setzen, nicht nur ähnliche Figuren zeichnen, sondern auch ähnliche kinematografische Inszenierungsweisen aufweisen. Darüber stellt sich beim Zuschauer ein ähnliches Verstehen her (das audiovisuelle Konstruieren eines Paares und eines Nebenbuhlers) sowie ein ähnliches Gefühl: das Genießen und die Lust zwischen den Einstellungen eine miteinander schwingende, subtile Bild-Dyade $\mathrm{zu}$ erleben. Es gibt einen Widerspruch zwischen dem narrativem Wissen über das ,offizielle Paar' (das vor allem durch den Dialog geprägt ist) und dem audiovisuell inszenierten ,eigentlichen Paar (welches im Wesentlichen durch Montage und Schauspiel entsteht), dies erzeugt ein Genießen, eine Lust am Sehen einer subtilen Verbindung, welche einen exklusiven Charakter aufweist.

Auf alle Kategorien bezogen kann man abstrahiert zusammenfassen: Die Filme generieren gemeinsam die Idee einer Paargemeinschaft, welche stets neu justiert werden muss, sie handelt nur zu einem geringeren Anteil von Liebe. Im Zentrum dieser Idee vom Paar steckt eine pragmatische Sicht von Beziehung. Dabei zeigen die Filme, wie Paargemeinschaften in den 1930er und 1940er Jahren zum Teil als durch finanzielle oder statusbezogene Interessen bestimmt imaginiert wurden. Dies geht soweit, dass sogar die Verhaltensformen, die in den Filmen ausgestellt werden, wie Tauschobjekte erscheinen, die instrumentell eingesetzt sich einlösen gegen andere Kommunikationen und Verhaltensweisen. Das Zwischenmenschliche wird ökonomisiert. Außerdem werden der soziale Raum, das familiäre Umfeld, aber auch die gesetzlichen Institutionen als einheitsstiftend dargestellt. Die Autoritäten überprüfen, strafen, mahnen und korrigieren das Paar, es kämpft aber auch dagegen an, indem es Rollen und Trugbilder spielt und darüber die gesellschaftliche Kontrolle umgeht. Das Paar ist ebenbürtig oder ringt darum, es zu sein. Das betrifft auch die Idee einer stark kontrollierenden Instanz, vor der sich das Paar rechtfertigt, vor der es spielt oder die es negiert.

Orientiert man sich an Cavells Konzept der Familienähnlichkeit, wie oben ausgeführt, dann kann man eine Entfaltung des Genres konkret an den Ausdrucksformen, ihren Kompositoriken sowie an der Art und Weise ihrer Bearbeitung von Themen beschreiben. Dabei ist die Wiederaufnahme, Variation, die gemeinsame Perspektive auf ein Thema zentral bzw. der Bezug zueinander, die familienähnliche Konstellation. ${ }^{140}$

140 Wie oben ausgeführt: „The idea is that the members of a genre share the inheritance of certain conditions, procedures and subjects and goals of compositions." Cavell 1981, S. 28. 
Trotz der vielfältigen literarischen und kulturgeschichtlichen Vorläufer gibt es also, folgt man Cavells Konzeption der Familienähnlichkeit, einen ersten Film, der etwas völlig Neues schafft: IT HAPPENED ONE NIGHT. ${ }^{141}$ Das Neue als Beginn des Genres meint nach Cavell die Reflexion bestimmter Themen sowie die (damit verbundene), sehr spezifische Ausdrucksform, welche der Film artikuliert. Es ist der Erfahrungsmodus, der durch filmische Expressivität geprägt, eine Genreformation ausmacht. Wie in den vorherigen Kapiteln erläutert, sind es die kompositorischen Muster, Inszenierungsweisen sowie die poetologischen Prinzipien der Screwball Comedy, welche das Genre prägen.

Das Screwball Genre beginnt mit einem Film, der völlig neue Formen beinhaltet, wie er das Medium nutzt. Dazu gehört die Art und Weise, wie die Rede mit den Montagesequenzen verwoben wird, wodurch ein Diskurs (im Fall von IT HAPPENED ONE NIGHT ist es das Zurechtkommen mit wenig Geld sowie die Schwierigkeit des Paares, sich zu einigen) über eineinhalb Stunden ausdifferenziert wird, indem bestimmte Elemente abgewogen, hervorgehoben oder verworfen werden. Dann taucht ein zweiter Film auf, der dem ersten gerade nicht in seiner Narration, aber in seiner Paarinszenierung ähnelt - dem Streit und den sich vermählenden Gesten (TWENTIETH CENTURY). Ein dritter Film (THE THIN MAN) festigt nun etwas an dem sich formierenden Genre; durch seine Kompositoriken beleuchteter eine Seite der vorherigen Filmen, die von einer zum Teil solidarischen, zum Teil höchst widersprüchlichen aber immer pragmatischen Gemeinschaft von Mann und Frau ausgeht. Diese Eigenschaft ebnet sich zu einer grundlegenden Konstellation vieler Filme des Genres. Mit MY MAN GODFREY erscheint ein Film, der seinen Fokus auf die große soziale Kluft zwischen dem Paar, die es zu überwinden gilt, setzt. In dieser Betonung lehnt der Film sich an den ersten, IT HAPPENED ONE NIGHT, an, in dem eine Millionärstochter sich in einen armen Journalisten verliebt. Diesmal verliebt sich eine Millionärstochter in einen Obdachlosen. Doch neben den rein narrativen Elementen wird mit MY MAN GODFREY auch eine weitere Dimension innerhalb des Genres in Gang gesetzt: Es ist eine Ähnlichkeit, die sich an den wiederkehrenden Schauspielern bemerkbar macht (was man mit Cavell „recurrent faces“142 nennen kann). Carole Lombards Schauspiel, ihre

141 Die meisten Arbeiten zum Genre der Screwball Comedy sind sich darüber einig, dass der Filmzyklus der Screwball Comedy mit IT HAPPENED ONE NIGHT begonnen habe. Der Filmzyklus wurde „screwball“, „madcap“, „daffy“ genannt. Balio (1995) nennt IT HAPPENED ONE NIGHT, TWENTIETH CENTURY und THE THIN MAN als Dreierpack, das beginnt, mit MR. DEED GOES TO TOWN seinen Höhepunkt erreiche und 1938 wieder versiege, Balio 1995, S. 268. THE THIN MAN (Woodbridge S. Van Dyke, USA 1934). MR. DEEDS GOES TO TOWN (Frank Capra, USA 1936).

142 Stanley Cavell: The World Viewed. Reflections on the Ontology of Film. Cambridge 1979, S. 75 . 
vibrierenden, exaltierten und gleichzeitig anmutig-eleganten Körperbewegungen, die schon in TWENTIETH CENTURY präsent waren, werden in ähnlicher Weise eingesetzt. An dieser Stelle kann man bereits zusammenfassen, wie sich eine konkrete Entfaltung des Genres nach dem Prinzip der Familienähnlichkeit ausgestaltet: Ein neuer Film, der erscheint, fügt dem Genre nicht einfach ein neues Element hinzu, sondern er schafft durch seine eigene Inszenierung zunächst einen bestimmten Schwerpunkt. Dieser hat etwas mit den ihm vorausgehenden Filmen zu tun, indem er eine bestimmte Seite an ihnen betont und ausfaltet. Durch die Inszenierung, z.B. die Kluft zwischen Arm und Reich in MY MAN GODFREY, wird nicht einfach nur ein Thema aus dem ersten Film IT HAPPENED ONE NIGHT wiederholt, sondern das Genre ändert sich in seinen Eigenschaften als Ganzes. Im Genre wird nun plötzlich stärker der Aspekt des Sozialen unterstrichen, es treten bestimmte Elemente auch der vorausgehenden Filme hervor. Die Obdachlosen in MY MAN GODFREY werden als solidarische Gemeinschaft inszeniert. Sie helfen einander aus, der eine bringt dem anderen eine Habseligkeit, sie unterstützen sich. Die dunkle, schattige Lichtinszenierung unterstützt, dass man die Männer kaum unterscheiden kann. Völlig konträr dazu wird die High Society dargestellt: In hellem Licht, alle Gegenstände glänzen und grenzen sich voneinander ab, trägt jeder sein ganz individuelles Kleid, man vergleicht sich.

Man braucht nicht das ganze Genre weiterzuverfolgen, um das Konzept der Familienähnlichkeit an den Filmen nachzuvollziehen; es sei hier nur das Prinzip verdeutlicht. Stets kommt ein neuer Film hinzu, dieser stellt eine eigene Weltwahrnehmung aus und mit dieser bezieht er sich reflexiv auf bestimmte gesellschaftliche Themen, aber auch auf Motive, Dramaturgien, Inszenierungsformen, Figuren, Schauspielweisen und Kompositionselemente vergangener Filme. Nicht alle Filme teilen alle Merkmale, aber jeder Film geht Beziehungen mit anderen Filmen ein, ist manchen ähnlicher als anderen. Die Ähnlichkeiten und Merkmale, welche sich verbinden, kann man nun gerade nicht über fixe oder abweichende Muster fassen, sondern über eine Linie von Familienähnlichkeiten, die sich thematisch, ästhetisch, motivisch, dramaturgisch, affektiv, expressiv und narrativ ausprägen.

Das Ende einer Filmreihe innerhalb eines Genres kann man als das beschreiben, was Cavell die Sättigung von Expressivität genannt hat. Es ändern sich die Stoffe, die Stimmung, die Konflikte. Freilich findet man viele Elemente der Screwball Comedy auch nach ADAM'S RIB oder I WAS A MALE WAR BRIDE wieder. ${ }^{143}$

143 Viele Genreelemente werden z.B. in Billy Wilders Filmen fortgesetzt. So lässt sich z.B. SOME LIKE IT HOT (Billy Wilder, USA 1959) als Remake von THE PALM BEACH STORY lesen. 
Davon grundverschieden sind jedoch die Gesellschafts- und Sex-Komödien der 1950er Jahre, die völlig neue Konstellationen zur Darstellung bringen und mit ihrer engen Ausrichtung auf die einzelne, kalkulierende Frau eine singuläre Figur zeichnen und keine Paareinheit mehr. Sie nehmen zwar Elemente des Screwball Genres auf - das Maskeradenspiel sowie die Emotionskarikatur - jedoch kreisen die Filme weniger um das Paar, als um die selbstbewusste SingleFrau oder die Gemeinschaft von Frauen mit Heiratswunsch (z.B. HOW TO MARRY A MILLIONAIRE $^{144}$ ).

Innerhalb der Filmgeschichte tauchen aber auch immer wieder Komödien auf, welche als direkte Nachkommen der Screwball Film gelten: WHAT'S UP DOC? ${ }^{145}$, THE CURSE OF THE JADE SCORPION ${ }^{146}$ oder INTOLERABLE CRUELTY ${ }^{147}$ sind wohl die eindeutigsten Beispiele einer späten Genreverwandtschaft. In einem größeren Zusammenhang ist wohl die Verwandtschaft zur Romantic Comedy am Auffälligsten: Das gesamte Genre ist durchsetzt von Elementen der klassischen Screwball Comedy. Jüngere Beispiele interpretieren dabei oftmals eine klassische Genre-Konstellation der Screwballs und machen sie zum HauptThema in der Dramaturgie. So kann man GHOSTS OF GIRLFRIENDS PAST ${ }^{148}$ direkt als Hommage an TOPPER ${ }^{149}$ verstehen, HOW TO LOSE A GUY IN 10 DAYS ${ }^{150}$ nimmt wiederum die Genrekategorie Maskerade, Bühne und des Spiels auf und dehnt sie auf einen ganzen Film aus. Anders als in der Screwball Comedy liegt der Fokus in der Romantic Comedy jedoch deutlich auf einem ausgeprägten Wechsel zwischen melodramatischer und komödischer Inszenierung.

\subsection{Ausklang}

In diesem Kapitel fokussierte ich eine makroanalytische Perspektive. Dabei standen zentrale Prinzipien des Genres im Zentrum. Zunächst wurde anhand von drei theoretischen Positionen die Zeitform der Komödie in den Blick genommen. Ein Ergebnis daraus ist, dass die Komödie als in ihrem Zeitmaß u.a. rasch, tempowechselnd, abrupt, überraschend und wiederholend qualifiziert wird. Von der Komödienerfahrung kommend wurde dann der Begriff der

\footnotetext{
144 HOW TO MARRY A MILLIONAIRE (Jean Negluesco, USA 1953).

145 WHAT'S UP, DOC? (Peter Bogdanovich, USA 1972).

146 THE CURSE OF THE JADE SCORPION (Woody Allen, USA 2001).

147 InTOLERABLE CRUELTY (Joel Coen und Ethan Coen, USA 2003).

148 GHOSTS OF GIRLFRIENDS PAST (Mark Waters, USA 2009).

149 TOPPER (Norman Z. McLeod, US, 1937).

150 HOW TO LOSE A GUY IN 10 DAYS (Donald Petrie, USA 2003).
} 
Familienähnlichkeit erörtert und in Bezug auf eine Entfaltung des Genres über die Zeit gesetzt. Im Anschluss daran standen verschiedene genrespezifische Kategorien im Zentrum, welche auf den Untersuchungsgegenstand bezogen ein Ergebnis der gesamten Arbeit darstellen. Sie bündeln die Beobachtungen, die aus den Analysen gezogen wurden, sie sind affektpoetisch, narrativ und thematisch. Die Kategorien denke ich als Teilelemente einer Idee, die alle Genrefilme gemeinsam reflektieren. Mit dieser Idee ist die Art und Weise gemeint, wie die Filme ein Denken anordnen. 